\title{
Secondary CMB anisotropies from the kinetic SZ effect
}

\author{
P. Valageas ${ }^{1}$, A. Balbi ${ }^{2}$, and J. Silk ${ }^{3,4}$ \\ 1 Service de Physique Théorique, CEA Saclay, 91191 Gif-sur-Yvette, France \\ 2 Dipartimento di Fisica, Università Tor Vergata, 00133 Roma, Italy \\ 3 Astrophysics, Department of Physics, Keble Road, Oxford OX1 3RH, UK \\ 4 Institut d'Astrophysique de Paris, CNRS, 98bis boulevard Arago, 75014 Paris, France
}

Received 28 July 2000 / Accepted 29 November 2000

\begin{abstract}
The reionization of the universe by stars and quasars is expected to be a highly inhomogeneous process. Moreover, the fluctuations of the matter density field also lead to inhomogeneities of the free electron distribution. These patterns gave rise to secondary CMB anisotropies through Thomson scattering of photons by free electrons. In this article we present an analytic model, based on our previous work which tackled the reionization history of the universe, which allows us to describe the generation of these secondary CMB anisotropies. We take into account the "patchy pattern" of reionization (HII bubbles), the cross-correlations of these ionized regions, the small-scale fluctuations of the matter density field and the contribution from collapsed objects. For an open universe, we find that the angular correlation function $C(\theta)$ displays a very slow decline from $C(0) \sim 610^{-13}$ up to the scale $\theta \sim 10^{-3}$ rad where it shows a sharp drop. On the other hand, the power-spectrum $l(l+1) C_{l} /(2 \pi)$ (and the "local average" $S_{l}$ ) exhibits a plateau of height $\sim 10^{-13}$ in the range $10^{3}<l<10^{6}$. We find that for large wavenumbers $l>10^{4}$ the signal is dominated by the contribution from collapsed halos while for $l<10^{4}$ it is governed by the large-scale correlations of HII bubbles. This implies that one cannot discriminate reionization by stars from a quasar-driven scenario since the size of ionized regions never dominates the behaviour of the anisotropies. Moreover, the secondary CMB anisotropies arise from a broad range of redshifts $(7.5<z<10$ for the IGM and $0<z<7$ for galactic halos). Thus, we find that the generation of these anisotropies involves several intricate processes and they are close to the resolution limit of current numerical simulations. The signal expected in our model might bias the cosmological parameter estimation from CMB experiments such as Planck or MAP, and could be detected by future mm-wavelength interferometers (e.g., ALMA).
\end{abstract}

Key words. cosmology: cosmic microwave background - cosmology: theory - galaxies: intergalactic medium cosmology: large-scale structure of Universe

\section{Introduction}

Observations of the spectra of distant quasars show that the universe is highly ionized by $z=5$, while recombination took place at $z \sim 1100$. In current cosmological scenarios, the reionization (and reheating) of the universe occurs at $z_{\text {ri }} \sim 10$ (typically $6 \lesssim z_{\text {ri }} \lesssim 15$ ) when structure formation is sufficiently advanced to build a large number of radiation sources (galaxies or quasars) which photoionize the IGM (e.g., Valageas \& Silk 1999a). However, the whole reionization history is a gradual and inhomogeneous process: each emitting object builds an HII region in its surroundings and reionization occurs when these bubbles overlap. This last stage is very rapid (e.g., Gnedin 2000) but at earlier redshifts there is a very inhomogeneous phase which evolves rather slowly, as the size of the ionized regions grows and the number of radiation

Send offprint requests to: P. Valageas, e-mail: valageas@spht.saclay.cea.fr sources increase. Then, this process can leave an imprint on the CMB through Thomson scattering of photons from free electrons. First, the mixing of photons coming from different lines of sight leads to a damping of small-scale primary fluctuations. Second, the Doppler effect (photons get some of the peculiar momentum of free electrons) generates secondary anisotropies since the distribution of free electrons is highly inhomogeneous. Thus, observations of CMB anisotropies could provide some information on the properties of the reionization process and on the features of the IGM at high redshifts.

As pointed out by Sunyaev (1978) and Kaiser (1984) the oscillations of the velocity field (as opposite sides of overdensities have almost opposite velocities) lead to a strong suppression of these secondary anisotropies. However, the modulation produced by the spatial variation of the number density of free electrons removes this cancelation on small scales and can generate significant CMB anisotropies. These fluctuations of the density of free 
electrons can be produced by several processes. First, as explained above, spatial variations of the ionized fraction of hydrogen due to patchy reionization provide a source of inhomogeneities (even if the IGM is uniform). This is relevant before reionization. Second, the fluctuations of the matter density field itself lead to inhomogeneities of the density of free electrons. This occurs both before and after reionization. When the density fluctuations are in the linear regime this corresponds to the Ostriker-Vishniac effect (Ostriker \& Vishniac 1986) while the non-linear regime is usually called the kinetic Sunyaev-Zel'dovich effect (e.g., Sunyaev \& Zel'dovich 1980).

In this article, we study both processes (patchy reionization and matter density fluctuations) in a unified fashion. To this order, we use an analytic model described in a previous paper (Valageas \& Silk 1999a) which we built to investigate the reionization and reheating history of the universe. It includes a model for galaxy formation (described in details in Valageas \& Schaeffer 1999) and for the quasar multiplicity function, which have been compared with observations at low redshifts $(z<4.5)$. Moreover, it also provides a description of the correlations of the matter density field which is consistent with these mass functions. The underlying model of the non-linear density field is based on the stable-clustering ansatz as detailed in Balian \& Schaeffer (1989) (see also Valageas \& Schaeffer 1997). This allows us to take into account density fluctuations within the IGM, the reionization process through the creation of HII regions and the correlations of these ionized bubbles. In addition to the IGM, we also consider the contribution from galactic halos. Here we restrict ourselves to the temperature anisotropies and we do not consider polarization. Thus, the main goals of this article are to:

- present an analytic model which can describe in a more detailed fashion than previous works the generation of these secondary CMB anisotropies. Moreover, our approach is self-consistent and it agrees with observations at low redshifts;

- obtain the redshift distribution of the contributions to these anisotropies;

- take advantage of the fact that we use an analytic model to explicitly separate the contributions from different physical processes (small-scale density fluctuations, patchy reionization, correlations of ionized bubbles). This allows us to see which information can be recovered from observations of the CMB;

- investigate whether one can discriminate reionization by stars versus quasars from the observed CMB anisotropies;

- check whether the main features of this process strongly depend on the cosmological scenario.

We present in Sect. 2 the formalism we use to compute these secondary CMB anisotropies, as well as the approximations we introduce. Then, in Sect. 3 we describe our numerical results for the case of an open universe. This corresponds to the model we used in a previous work (Valageas \& Silk 1999a) to investigate the reionization history of the universe. In the last section we also consider briefly a critical density universe for comparison.

\section{Secondary anisotropies}

\subsection{Contribution from inhomogeneities of the free electron distribution}

As described for instance in Gruzinov \& $\mathrm{Hu}$ (1998) and Knox et al. (1998), Thomson scattering of CMB photons off moving free electrons in the IGM generates secondary anisotropies. Thus, for small optical depths the temperature perturbation $\Delta_{\mathrm{T}}(\hat{\gamma})=\delta T / T$ on the direction $\hat{\gamma}$ on the sky is:

$$
\begin{aligned}
\Delta_{\mathrm{T}}(\hat{\gamma}) & =-\int \mathrm{d} \boldsymbol{l} \cdot \frac{\boldsymbol{v}}{c} \sigma_{\mathrm{T}} n_{\mathrm{e}, \mathrm{f}} \mathrm{e}^{-\tau} \\
& =-\frac{\sigma_{\mathrm{T}} c}{H_{0}} \int \frac{\mathrm{d} \chi}{1+z} \frac{\hat{\boldsymbol{\gamma}} \cdot \boldsymbol{v}}{c} n_{\mathrm{e}, \mathrm{f}}
\end{aligned}
$$

where $\sigma_{\mathrm{T}}$ is the Thomson cross-section, $n_{\mathrm{e}, \mathrm{f}}$ the number density of free electrons, $\boldsymbol{v}$ the peculiar velocity and $l$ the coordinate along the line of sight, all in physical units. We also defined $\chi$ as the dimensionless radial comoving coordinate:

$\mathrm{d} \chi=\frac{\mathrm{d} z}{\sqrt{\Omega_{\Lambda}+\left(1-\Omega_{\mathrm{m}}-\Omega_{\Lambda}\right)(1+z)^{2}+\Omega_{\mathrm{m}}(1+z)^{3}}}$.

Since in our scenario reionization occurs at $z=6.8$, the optical depth $\tau$ from $\chi=0$ up to $\chi$ in (1) is very small $(\tau<0.024$, see Fig. 15 in Valageas \& Silk 1999a) and it plays no role so that we used the approximation $\mathrm{e}^{-\tau} \simeq$ 1. Then, the two-point correlation function $C(\theta)$ of these temperature distortions is simply:

$$
\begin{aligned}
C(\theta)= & \left(\frac{\sigma_{\mathrm{T}} c}{H_{0}}\right)^{2} \int \frac{\mathrm{d} \chi_{1}}{1+z_{1}} \int \frac{\mathrm{d} \chi_{2}}{1+z_{2}} \\
& \times\left\langle\frac{\hat{\gamma}_{1} \cdot \boldsymbol{v}_{1}}{c} \frac{\hat{\boldsymbol{\gamma}}_{2} \cdot \boldsymbol{v}_{2}}{c} n_{\mathrm{e}, \mathrm{f}, 1} n_{\mathrm{e}, \mathrm{f}, 2}\right\rangle
\end{aligned}
$$

where the directions $\hat{\gamma}_{1}$ and $\hat{\gamma}_{2}$ make the angle $\theta$. One can distinguish two effects which contribute to the correlation function in (3). Firstly, a uniform reionization in a homogeneous IGM (i.e. $n_{\mathrm{e}, \mathrm{f}, 1}$ shows no fluctuations) provides a non-zero contribution through the fluctuations $\left\langle\left(\hat{\gamma}_{1} \cdot \boldsymbol{v}_{1}\right)\left(\hat{\gamma}_{2} \cdot \boldsymbol{v}_{2}\right)\right\rangle$ of the velocity field. However, because of the oscillations of the velocity correlation (related to the infall from opposite sides into potential wells) the integration along the line of sight leads to a strong suppression of these Doppler effects (Sunyaev 1978; Kaiser 1984). Secondly, the inhomogeneities of the free electron number density add a second contribution which avoids this cancelation of the velocity term. Most of the power which builds the fluctuations of the velocity comes from scales of the order of $R_{-1}$ where the local slope of the power-spectrum is $n=-1$. This gives $R_{-1} \sim 10$ comoving Mpc. On the other hand, the characteristic scale of the inhomogeneities of the electron distribution is of order $R_{n_{\mathrm{e}}} \sim 100$ comoving kpc (it is set by the size of the ionized bubbles and the 
scale of non-linear structures). Thus, we make the approximation that the velocity fluctuations are not correlated to the density field and we write:

$$
\begin{aligned}
C(\theta)= & \tau_{0}^{2} \int \mathrm{d} \chi_{1} \mathrm{~d} \chi_{2}\left(1+z_{1}\right)^{2}\left(1+z_{2}\right)^{2}\left\langle\frac{\hat{\gamma}_{1} \cdot \boldsymbol{v}_{1}}{c} \frac{\hat{\boldsymbol{\gamma}}_{2} \cdot \boldsymbol{v}_{2}}{c}\right\rangle \\
& \times\left[\left\langle\left(1+\delta_{1}\right) x_{\mathrm{e}, 1}\left(1+\delta_{2}\right) x_{\mathrm{e}, 2}\right\rangle\right. \\
& \left.-\left\langle\left(1+\delta_{1}\right) x_{\mathrm{e}, 1}\right\rangle\left\langle\left(1+\delta_{2}\right) x_{\mathrm{e}, 2}\right\rangle\right]
\end{aligned}
$$

where $x_{\mathrm{e}}=n_{\mathrm{e}, \mathrm{f}} / n_{\mathrm{e}}$ is the ionization fraction, $\delta=\left(n_{\mathrm{b}}-\right.$ $\left.\bar{n}_{\mathrm{b}}\right) / \bar{n}_{\mathrm{b}}$ is the density contrast and we defined:

$\tau_{0}=\frac{n_{\mathrm{e}, 0} \sigma_{\mathrm{T}} c}{H_{0}}$.

Here $n_{\mathrm{e}, 0}$ is the present number density of free electrons:

$n_{\mathrm{e}, 0}=\frac{\Omega_{\mathrm{b}}}{\Omega_{\mathrm{m}}} \frac{\bar{\rho}_{0}}{m_{\mathrm{p}}}\left(1-\frac{Y}{2}\right)$

where $Y=0.26$ is the helium mass fraction. The difference which enters the term in the second line of expression (4) corresponds to the fact that here we only consider the contribution to secondary CMB anisotropies due to the modulation of the velocity field by the fluctuations of the free electron number density. Thus, for a uniform free electron density field the quantity $C(\theta)$ written in (4) vanishes. As we discussed above, the factorization of the velocity and density averages we used in (4) is valid at small scales $r \ll R_{-1}$ where we actually have: $\left\langle\left(\hat{\gamma}_{1} \cdot \boldsymbol{v}_{1}\right)\left(\hat{\gamma}_{2} \cdot \boldsymbol{v}_{2}\right)\right\rangle \simeq\left\langle\left(\hat{\gamma}_{1} \cdot \boldsymbol{v}_{1}\right)\left(\hat{\gamma}_{2} \cdot \boldsymbol{v}_{1}\right)\right\rangle \simeq\left\langle\left(\hat{\gamma}_{1} \cdot \boldsymbol{v}_{1}\right)^{2}\right\rangle$. To get the last approximation we used the fact that the lines of sight $\hat{\gamma}_{1}$ and $\hat{\gamma}_{2}$ must be nearly parallel since all scales of interest are much smaller than the Hubble scale. In other words, at small distances we can consider the velocity field to be constant. However, in practice we shall also consider larger scales where the cross-correlation of ionized bubbles generates some power. These scales are close to $R_{-1}$ hence we keep the expression (4) rather than making the approximation $\left\langle\left(\hat{\gamma}_{1} \cdot \boldsymbol{v}_{1}\right)\left(\hat{\gamma}_{2} \cdot \boldsymbol{v}_{2}\right)\right\rangle \simeq\left\langle v^{2}\right\rangle / 3$. However, on these scales we can expect to underestimate somewhat the angular correlation $C(\theta)$ since the velocity field should be correlated with the density field. We note $\xi_{\mathrm{v}}\left(\boldsymbol{R}_{\theta}+\boldsymbol{l}\right)$ the velocity term and $\xi_{\mathrm{e}}\left(\boldsymbol{R}_{\theta}+\boldsymbol{l}\right)$ the electron density correlation which enter the expression (4) and we write (4) as:

$C(\theta)=\tau_{0}^{2} \int \mathrm{d} \chi \frac{H_{0}}{c}(1+z)^{5} \int_{-\infty}^{\infty} \mathrm{d} l \xi_{\mathrm{v}}\left(\boldsymbol{R}_{\theta}+\boldsymbol{l}\right) \xi_{\mathrm{e}}\left(\boldsymbol{R}_{\theta}+\boldsymbol{l}\right) \cdot(7)$

Here $l$ is the physical length along the line of sight while $\boldsymbol{R}_{\theta}$ is the physical distance at redshift $z$ between both lines of sight $\left(\boldsymbol{R}_{\theta}\right.$ is orthogonal to $\left.\boldsymbol{l}\right)$ :

$R_{\theta}(z)=\frac{c}{H_{0}} \frac{\mathcal{D}(z)}{1+z} \theta$

where the dimensionless comoving angular distance $\mathcal{D}$ is defined by:

$\mathcal{D}(z)=\frac{1}{\sqrt{1-\Omega_{\mathrm{m}}-\Omega_{\Lambda}}} \sinh \left(\sqrt{1-\Omega_{\mathrm{m}}-\Omega_{\Lambda}} \chi\right)$.
In this article we only consider the secondary anisotropies due to the inhomogeneities of the free electron number distribution, described by (7). As discussed above, the contribution which arises from the fluctuations of the velocity field only is smaller because of the cancelations along the line of sight.

\subsection{Velocity fluctuations}

First, the velocity term in (7) is obtained as follows. Since most of the scales which contribute to the velocity fluctuations are in the linear regime until $z=0$, we can use the linear relation between the velocity and density fluctuations (Peebles 1980):

$\boldsymbol{v}(\boldsymbol{x})=a H(z) f\left[\Omega_{\mathrm{m}}(z)\right] \int \mathrm{d}^{3} k \mathrm{e}^{i \boldsymbol{k} \cdot x} \frac{i \boldsymbol{k}}{k^{2}} \delta(\boldsymbol{k})$

where $f\left(\Omega_{\mathrm{m}}\right) \simeq \Omega_{\mathrm{m}}^{0.6}$ if $\Omega_{\Lambda}=0, a$ is the scale factor $(a=1$ at $z=0), \boldsymbol{k}$ and $\boldsymbol{x}$ are comoving quantities. Since we consider lines of sight which are almost parallel (small angles $\theta$ ) we write the velocity correlation as (e.g., Groth et al. 1989):

$$
\begin{aligned}
\xi_{\mathrm{v}}(\boldsymbol{x})= & \frac{4 \pi}{c^{2}}\left(a H(z) f\left[\Omega_{\mathrm{m}}(z)\right]\right)^{2} \int_{0}^{\infty} \mathrm{d} k P(k ; z) \\
& \times\left[j_{0}(k x)-2 \frac{j_{1}(k x)}{k x}\right]
\end{aligned}
$$

where $j_{0}$ and $j_{1}$ are the spherical Bessel functions. Here we introduced the linear power-spectrum defined by $\left\langle\delta\left(\boldsymbol{k}_{1}\right) \delta\left(\boldsymbol{k}_{2}\right)\right\rangle=P\left(k_{1}\right) \delta_{\mathrm{D}}\left(\boldsymbol{k}_{1}+\boldsymbol{k}_{2}\right)$. Next we define at $z=0$ :

$v_{0}^{2}=4 \pi\left(H_{0} f\left(\Omega_{\mathrm{m}, 0}\right)\right)^{2} \int_{0}^{\infty} \mathrm{d} k P(k ; 0)$

and

$\Pi_{\mathrm{v}}(x)=3 \frac{\int \mathrm{d} k P(k ; 0)\left[j_{0}(k x)-2 \frac{j_{1}(k x)}{k x}\right]}{\int \mathrm{d} k P(k ; 0)}$.

Thus, $\Pi_{\mathrm{V}}(x)$ only depends on the shape of the powerspectrum and we have $\Pi_{\mathrm{v}}(0)=1$ while at large scales $x \gg R_{-1}$ it shows an oscillatory behaviour. Then, we can write $\xi_{\mathrm{v}}(\boldsymbol{x})$ as:

$\xi_{\mathrm{v}}(\boldsymbol{x})=\frac{v_{0}^{2}}{3 c^{2}} \Pi_{\mathrm{v}}(x)\left[(1+z)^{-2} \frac{H(z)}{H_{0}} \frac{f\left[\Omega_{\mathrm{m}}(z)\right]}{f\left(\Omega_{\mathrm{m}, 0}\right)}\right]^{2}$

where we used the redshift evolution $P(k) \propto(1+z)^{-2}$ of the linear power-spectrum.

\subsection{Free electron density fluctuations}

Second, we have to model the fluctuations of the free electron density distribution. As seen in (4) two effects contribute to these fluctuations: i) inhomogeneities of the baryonic matter distribution and ii) of the ionization fraction. In most previous studies of these secondary CMB anisotropies only the second contribution was taken into 
account through a model of ionized bubbles within a uniform IGM (e.g., Gruzinov \& Hu 1998; Knox et al. 1998). However, as shown in Valageas \& Silk (1999a) the clumping of the gas is not negligible even at $z \sim 10$. As can be seen from (4), and as we shall check below, this increases somewhat the amplitude of these CMB anisotropies. In this article we use a simple model to estimate the correlation term $\xi_{\mathrm{e}}(r)$. First, although the baryonic density fluctuations may be correlated with the ionization fraction we make the approximation:

$\xi_{\mathrm{e}} \simeq\left\langle\left(1+\delta_{1}\right)\left(1+\delta_{2}\right)\right\rangle\left\langle x_{\mathrm{e}, 1} x_{\mathrm{e}, 2}\right\rangle-\langle 1+\delta\rangle^{2}\left\langle x_{\mathrm{e}}\right\rangle^{2}$

which can be written:

$\xi_{\mathrm{e}}(r)=\left(\left[1+\xi_{\mathrm{IGM}}(r)\right]\left\langle x_{\mathrm{e}, 1} x_{\mathrm{e}, 2}\right\rangle(r)-\left\langle x_{\mathrm{e}}\right\rangle^{2}\right)\langle 1+\delta\rangle_{\mathrm{IGM}}^{2} \cdot$

Here we note $\xi_{\mathrm{IGM}}(r)$ the two-point correlation function of the gas density within the IGM and $\langle\delta\rangle_{\text {IGM }}$ the mean density contrast of the IGM. Note that at low redshifts after reionization $x_{\mathrm{e}}=1$ almost everywhere within the IGM and the relation $\xi_{\mathrm{e}}(r)=\xi_{\mathrm{IGM}}(r)\langle 1+\delta\rangle_{\mathrm{IGM}}^{2}$ becomes exact. Until $z \sim 1$ most of the matter is contained in the IGM since only a very small amount of gas was able to cool and form galaxies, hence $\langle 1+\delta\rangle_{\text {IGM }}$ is close to unity, while at $z=0$ we have $\langle 1+\delta\rangle_{\text {IGM }} \sim 0.4$ (Valageas \& Silk 1999a; Valageas et al. 2000).

\subsubsection{Ionization fraction}

In order to obtain the term $\left\langle x_{\mathrm{e}, 1} x_{\mathrm{e}, 2}\right\rangle(r)$ we use a model of spherical ionized bubbles around galaxies and quasars as in Valageas \& Silk (1999a). Thus, we consider that $x_{\mathrm{e}}=1$ within ionized patches and $x_{\mathrm{e}}=0$ everywhere else. At low $z$ after overlap of the ionized regions $x_{\mathrm{e}}=1$ throughout the IGM. Indeed, reionization occurs thanks to the growth of the ionized bubbles which finally occupy all the volume (and not through a slow increase of a uniform ionization fraction). Then, $\left\langle x_{\mathrm{e}, 1} x_{\mathrm{e}, 2}\right\rangle(r)$ is simply the probability that two points at distance $r$ are located within ionized regions. First, let us consider uncorrelated ionizing sources. Then, the probability that the first point $\boldsymbol{r}_{1}$ is within an ionized bubble is the volume fraction occupied by these regions:

$\mathcal{P}_{1}=\int \frac{\mathrm{d} x_{1}}{x_{1}} \frac{\bar{\rho}}{M_{1}} x_{1}^{2} H\left(x_{1}\right) V_{\text {ion }}\left(x_{1}\right)$

where $V_{\text {ion }}\left(x_{1}\right)$ is the volume of the ionized bubble associated to the source $x_{1}$ (galaxy or quasar) and $\left(\bar{\rho} / M_{1}\right) x_{1} H\left(x_{1}\right) \mathrm{d} x_{1}$ is the number density of radiation sources labelled by the parameter $x_{1}$ in the range $\left[x_{1}, x_{1}+\right.$ $\mathrm{d} x_{1}$ ] (see Valageas \& Silk 1999a; Valageas \& Schaeffer 1997). Next, a simple geometrical calculation shows that the second point $\boldsymbol{r}_{2}=\boldsymbol{r}_{1}+\boldsymbol{r}$ can be located within the same ionized spherical bubble of radius $R_{1}$ with a probability $\mathcal{P}_{2, \mathrm{~s}}$ :

$\mathcal{P}_{2, \mathrm{~s}}=F\left(\frac{r}{R_{1}}\right)$ with $\left\{\begin{array}{l}F(x)=1-\frac{3 x}{4}+\frac{x^{3}}{16} \text { if } x<2 \\ F(x)=0 \text { if } x \geq 2 .\end{array}\right.$
On the other hand, the point $\boldsymbol{r}_{2}$ can be embedded within another bubble with a probability $\mathcal{P}_{2, \mathrm{o}}$ with:

$\mathcal{P}_{2, \mathrm{o}}=\left[1-F\left(\frac{r}{R_{1}}\right)\right] \int \frac{\mathrm{d} x_{2}}{x_{2}} \frac{\bar{\rho}}{M_{2}} x_{2}^{2} H\left(x_{2}\right) V_{\text {ion }}\left(x_{2}\right)$

where we assumed that the bubbles associated to different sources do not overlap. This should be a good approximation before reionization when there are very few ionized regions. Hence we obtain for uncorrelated sources $\left\langle x_{\mathrm{e}, 1} x_{\mathrm{e}, 2}\right\rangle(r)=\mathcal{P}_{1}\left(\mathcal{P}_{2, \mathrm{~s}}+\mathcal{P}_{2, \mathrm{o}}\right)$. However, the sources $x_{1}$ and $x_{2}$ should be correlated, especially at high $z$ where they correspond to very rare large density fluctuations. Thus, we write:

$$
\begin{aligned}
& \mathcal{P}_{2}\left(r ; x_{1}\right)=\operatorname{Min}\left\{1, F\left(\frac{r}{R_{1}}\right)+\left[1-F\left(\frac{r}{R_{1}}\right)\right]\right. \\
&\left.\times \int \frac{\mathrm{d} x_{2}}{x_{2}} \frac{\bar{\rho}}{M_{2}} x_{2}^{2} H\left(x_{2}\right) V_{\text {ion }}\left(x_{2}\right)\left[1+\xi_{x_{1}, x_{2}}(r)\right]\right\}
\end{aligned}
$$

where $\xi_{x_{1}, x_{2}}(r)$ is the correlation function of the objects $x_{1}$ and $x_{2}$. Following the analytic results obtained in Bernardeau \& Schaeffer $(1992,1999)$ we have:

$\xi_{x_{1}, x_{2}}(r)=b\left(x_{1}\right) b\left(x_{2}\right) \xi(r)$

with

$x \ll 1: b(x) \propto x^{(1-\omega) / 2} \quad$ and $\quad x \gg 1: b(x) \propto x$

where $b(x)$ is the bias associated to an object defined by the parameter $x$. This behaviour has been shown to agree with the results of numerical simulations in Munshi et al. (1999). In Valageas et al. (2000) the predictions of this model for the correlation functions of galaxies, quasars, Lyman- $\alpha$ clouds and clusters have been compared to observations. Thus, this provides a unified model for all these objects (which are characterized by different scales and densities) which agrees reasonably well with observations and simulations. In (20) the use of the minimum ensures that $\mathcal{P}_{2} \leq 1$. Indeed, at small redshift after reionization the second term in the minimum of (20) becomes larger than unity since all the volume is ionized (the ionized bubbles overlap and $V_{\text {ion }}$ goes to infinity). Finally, we obtain $\left\langle x_{\mathrm{e}, 1} x_{\mathrm{e}, 2}\right\rangle(r)$ as:

$$
\begin{aligned}
& \left\langle x_{\mathrm{e}, 1} x_{\mathrm{e}, 2}\right\rangle(r)= \\
& \quad \operatorname{Min}\left\{1, \int \frac{\mathrm{d} x_{1}}{x_{1}} \frac{\bar{\rho}}{M_{1}} x_{1}^{2} H\left(x_{1}\right) V_{\text {ion }}\left(x_{1}\right) \mathcal{P}_{2}\left(r ; x_{1}\right)\right\}
\end{aligned}
$$

while the mean ionization fraction is simply:

$\left\langle x_{\mathrm{e}}\right\rangle=\operatorname{Min}\left\{1, \int \frac{\mathrm{d} x_{1}}{x_{1}} \frac{\bar{\rho}}{M_{1}} x_{1}^{2} H\left(x_{1}\right) V_{\text {ion }}\left(x_{1}\right)\right\}$.

The minima in (23) and (24) ensure again that $\left\langle x_{\mathrm{e}, 1} x_{\mathrm{e}, 2}\right\rangle \leq$ 1 and $\left\langle x_{\mathrm{e}}\right\rangle \leq 1$ after reionization. 


\subsubsection{Density fluctuations}

Finally, we need to evaluate the two-point correlation function $\xi_{\mathrm{IGM}}(r)$ of the gas density within the IGM which appears in (16). We consider that the universe is made of collapsed objects which have been able to cool and to form galaxies, embedded within a lower density medium which we call the IGM. Hence, the latter corresponds to voids as well as to density fluctuations (which may appear as filaments or shallow spherical halos) associated with the Lyman- $\alpha$ forest (Valageas et al. 1999). Then, as in Valageas \& Silk (1999a) the mean density of the matter which forms the IGM is given by:

$\langle 1+\delta\rangle_{\mathrm{IGM}}=(1+\Delta)_{\mathrm{u}}+\int_{0}^{x_{\mathrm{cool}}} \frac{\mathrm{d} x}{x} x^{2} H(x)$

while the mean square density is:

$\left\langle(1+\delta)^{2}\right\rangle_{\mathrm{IGM}}=(1+\Delta)_{\mathrm{u}}^{2}+\int_{0}^{x_{\mathrm{cool}}} \frac{\mathrm{d} x}{x} x^{2} H(x)(1+\Delta) .(26)$

The term $(1+\Delta)_{u}$ corresponds to voids while the second term in the rhs in (25) and (26) is the contribution from Lyman- $\alpha$ forest clouds. The upper bound $x_{\text {cool }}$ ensures that we do not count cooled objects (which are identified to galactic halos) nor clusters of galaxies, since they are not part of the IGM. Then, the clumping factor of the IGM is simply:

$C_{\mathrm{IGM}} \equiv 1+\xi_{\mathrm{IGM}}(r=0)=\frac{\left\langle(1+\delta)^{2}\right\rangle_{\mathrm{IGM}}}{\langle 1+\delta\rangle_{\mathrm{IGM}}^{2}}$

(which we noted $C_{\mathrm{n}}$ in Valageas \& Silk 1999a). In addition, as described in Valageas et al. (1999) and Valageas \& Silk (1999a), baryonic density fluctuations within the IGM are erased on small scales below $R_{\mathrm{d}}(z)$ due to the non-zero temperature $T_{\mathrm{IGM}}$ with:

$R_{\mathrm{d}}(z) \sim t_{\mathrm{H}} \sqrt{\frac{k T_{\mathrm{IGM}}}{\mu \mathrm{m}_{\mathrm{p}}}} \sim t_{\mathrm{H}} c_{\mathrm{s}}$.

This corresponds to the scale reached by acoustic waves over the Hubble time $t_{\mathrm{H}}$ (with the sound velocity $c_{\mathrm{s}}$ ). Note that the damping scale $R_{\mathrm{d}}$ is usually smaller than the standard Jeans scale $R_{\text {Jeans }}$ which corresponds to the limit of large times. Hence we write:

$r \leq R_{\mathrm{d}}(z): \quad \xi_{\mathrm{IGM}}(r)=\xi_{\mathrm{IGM}}\left(R_{\mathrm{d}}\right)=C_{\mathrm{IGM}}-1$

in order to take into account the damping of the baryonic power-spectrum on small scales within the IGM, in a fashion which is consistent with (27). Finally, for larger scales we use the prescription:

$r>R_{\mathrm{d}}(z): \quad \xi_{\mathrm{IGM}}(r)=\left(C_{\mathrm{IGM}}-1\right) \frac{\xi(r)}{\xi\left(R_{\mathrm{d}}\right)}$.

Note that this large $-r$ behaviour (i.e. for $r>R_{\mathrm{d}}$ ) is consistent with the factorization (21).

\subsection{Contribution from galactic halos}

In addition to the free electron IGM number density correlation function $\xi_{\mathrm{e}}(r)$ which we defined in (16), we also introduce the correlation function $\xi_{\mathrm{e}, \mathrm{gal}}(r)$ due to collapsed halos which were able to cool and to form galaxies. We estimate this contribution as follows. We assume that within these halos the gas is totally ionized (by the radiation of the central stars or QSO and by collisional ionization, due to shock-heating up to the virial temperature). Then, we can write (15) as:

$\xi_{\mathrm{e}, \mathrm{gal}}(r)=F_{\text {gal,vol }}^{2}\left(1+\Delta_{\mathrm{c}}\right)^{2} \xi_{\text {gal }}(r)=F_{\text {gal }}^{2} \xi_{\text {gal }}(r)$

where $F_{\text {gal,vol }}$ is the volume fraction (i.e. the filling factor) occupied by these objects and $F_{\text {gal }}=F_{\text {gal,vol }}\left(1+\Delta_{\mathrm{c}}\right)$ is the fraction of matter they contain. We note $\Delta_{c}(z)$ the mean density contrast of just-virialized halos, given by the usual spherical model, while $\xi_{\text {gal }}(r)$ is the matter correlation function associated with these halos. If we do not take into account the density profile of these objects (i.e. we take a constant density equal to $\left.\left(1+\Delta_{c}\right) \bar{\rho}\right)$ we have for the clumping of the gas:

$C_{\text {gal }}=1+\xi_{\text {gal }}(r=0)=\frac{1+\Delta_{c}}{F_{\text {gal }}} \gg 1$.

Then, in a fashion similar to (29) and (30) we write the matter correlation function as:

$r>R_{\text {cool }}(z): \quad \xi_{\text {gal }}(r)=\left(C_{\text {gal }}-1\right) \frac{\xi(r)}{\xi\left(R_{\text {cool }}\right)}$

and $\xi_{\text {gal }}(r)=\left(C_{\text {gal }}-1\right)$ for $r<R_{\text {cool }}$. Here we defined $R_{\text {cool }}(z)$ as the virial radius of the smallest halos which are able to cool at redshift $z$, as in Valageas \& Silk (1999a). Of course, our prescription for $\xi_{\mathrm{e}, \text { gal }}(r)$ is only meant to provide a crude estimate of the contribution due to galactic halos. Note that if we consider a specific density profile for the dark matter halos we would only need to multiply $C_{\text {gal }}$ by a numerical factor of order unity, provided that the slope of the density in the inner regions is shallower than $-3 / 2$ (otherwise $C_{\text {gal }}$ is not finite). In addition, one should take into account the additional collapse of the gas (while it cools) and the effects of matter ejection through supernovae or stellar winds. In this article, we shall restrict ourselves to the simple prescription (33) which should exhibit the right trend with $r$, keeping in mind that at small scales $r<R_{\text {cool }}$ we might underestimate the signal by a factor $\sim 3$. The comparison of $\xi_{\text {e,gal }}$ with $\xi_{\mathrm{e}}$ allows us to see which of these two contributions (from galactic halos or from the IGM) is dominant at a given scale. It also shows the characteristic scales associated with both processes.

\subsection{Power spectrum of secondary anisotropies}

Using the results of the previous sections, we can write the two-point correlation function $C(\theta)$ obtained in (7) as:

$C(\theta)=\frac{2 \tau_{0}^{2} v_{0}^{2}}{3 c^{2}} \int \mathrm{d} z w(z) \int_{R_{\theta}}^{\infty} \frac{\mathrm{d} r}{R_{z}} \frac{r \Pi_{\mathrm{v}}[r(1+z)] \xi_{\mathrm{e}}(r)}{\sqrt{r^{2}-R_{\theta}^{2}}}(34)$ 
with:

$w(z)=\frac{\mathrm{d} \chi}{\mathrm{d} z} \mathcal{D}(z)\left(\frac{H(z) f\left[\Omega_{\mathrm{m}}(z)\right]}{H_{0} f\left(\Omega_{\mathrm{m}, 0}\right)}\right)^{2}$

and:

$R_{z}=\frac{c}{H_{0}} \frac{\mathcal{D}(z)}{1+z}$

In (34) we made the change of variable $r=\left|\boldsymbol{R}_{\theta}+\boldsymbol{l}\right|$. The angular power-spectrum of these secondary anisotropies is given by:

$C_{l}=2 \pi \int \mathrm{d}(\cos \theta) P_{l}(\cos \theta) C(\theta)$

which for $l \gg 1$ can be approximated by:

$l \gg 1: \quad C_{l}=2 \pi \int_{0}^{\infty} \mathrm{d} \theta \theta J_{0}(l \theta) C(\theta)$

where $P_{l}$ are the Legendre polynomials and $J_{0}$ is the Bessel function of order 0. Then, from (34) we obtain after integration over $\theta$ (see Gradshteyn \& Ryzhik 1965, Sect. 6.554.2, p. 706):

$$
\begin{aligned}
C_{l}= & \frac{4 \pi \tau_{0}^{2} v_{0}^{2}}{3 c^{2} l} \int \mathrm{d} z w(z) \int_{0}^{\infty} \frac{\mathrm{d} r}{R_{z}} \frac{r}{R_{z}} \sin \left(\frac{l r}{R_{z}}\right) \\
& \times \Pi_{\mathrm{v}}[r(1+z)] \xi_{\mathrm{e}}(r) .
\end{aligned}
$$

The expression (39) clearly shows that $C_{l}$ will exhibit a strong decline at large $l$ for wavelengths which are smaller than the typical scales of the free electron inhomogeneities $\left(R_{z} / l \ll R_{n_{\mathrm{e}}}\right)$ because of the oscillatory term $\sin \left(l . r / R_{z}\right)$. On the other hand, for small $l$ (i.e. at large scales $R_{z} / l \gg R_{n_{\mathrm{e}}}$ where the correlation of the electron distribution vanishes) we recover a white noise powerspectrum ( $C_{l}$ is constant).

Finally, following Bruscoli et al. (2000) we define the quantity $S_{l}$ by:

$S_{l}=\frac{1}{2 \pi} \int_{l / 2}^{3 l / 2} \mathrm{~d} l l C_{l} \sim\left\langle\frac{l(l+1) C_{l}}{2 \pi}\right\rangle_{l}$.

This "local average" of $l(l+1) C_{l} /(2 \pi)$ removes some of the oscillations of $C_{l}$ at large $l$ and it allows us to see more clearly the drop at large $l$ of the spectrum. It is also more convenient for numerical calculations since it requires a lower resolution. From the relation (39) we obtain:

$$
\begin{aligned}
S_{l}= & \frac{2 \tau_{0}^{2} v_{0}^{2}}{3 c^{2}} \int \mathrm{d} z w(z) \int_{0}^{\infty} \frac{\mathrm{d} r}{R_{z}}\left(\cos \frac{l r}{2 R_{z}}-\cos \frac{3 l r}{2 R_{z}}\right) \\
& \times \Pi_{\mathrm{v}}[r(1+z)] \xi_{\mathrm{e}}(r) .
\end{aligned}
$$

Using (38) we can also write $S_{l}$ in terms of $C(\theta)$ as:

$S_{l}=\int_{0}^{\infty} \frac{\mathrm{d} \theta}{\theta} C(\theta)\left[\frac{3 l \theta}{2} J_{1}\left(\frac{3 l \theta}{2}\right)-\frac{l \theta}{2} J_{1}\left(\frac{l \theta}{2}\right)\right]$.

Note that the expressions (34), (39) and (41) are quite general and do not depend on our model for the inhomogeneities of the free electron distribution (which enters $\left.\xi_{\mathrm{e}}(r)\right)$. They merely use the linear evolution (14) of the velocity fluctuations and the approximation that velocity and density fluctuations are uncorrelated. Moreover, it is more accurate (hence more convenient in terms of numerical resolution) to compute $C_{l}$ and $S_{l}$ from (39) and (41) rather than from the Fourier transform of the final angular correlation $C(\theta)$.

\section{Numerical results}

For the numerical calculations we consider an open CDM universe (OCDM) with $\Omega_{\mathrm{m}}=0.3, \Omega_{\Lambda}=0, \Omega_{\mathrm{b}}=0.03$, $H_{0}=60 \mathrm{~km} \mathrm{~s}^{-1} / \mathrm{Mpc}$ and $\sigma_{8}=0.77$. These values are those we used in previous articles where we considered the luminosity functions of galaxies (Valageas \& Schaeffer 1999), Lyman- $\alpha$ absorbers (Valageas et al. 1999), clusters (Valageas \& Schaeffer 2000) and reionization by stars and quasars (Valageas \& Silk 1999a,b).

\subsection{Velocity fluctuations}

First, we display in Fig. 1 the magnitude of the velocity correlation function $\xi_{\mathrm{v}}(x)$ at $z=0$. It shows a plateau at small scales $x \ll R_{-1}$ (most of the power comes from $R_{-1}$ where the quantity $k P(k)$ is maximum) and it declines at large scales with oscillations. Thus, in our model velocity fluctations are of order $\Delta v \sim 400 \mathrm{~km} \mathrm{~s}^{-1}$ at $z=0$ (and they roughly decrease as $\left.(1+z)^{-1 / 2}\right)$. They only arise from the linear growth of initial perturbations through gravitational interaction. In the actual universe, an additional source of velocity fluctuations (mainly at small scales) could be provided by other processes like the ejection of matter by supernovae or turbulence. If these velocity fluctuations are larger by a factor $\alpha$ than the value we use for $\Delta v$ shown in Fig. 1, then on the corresponding scales we should roughly increase our predictions for the secondary CMB anistropies $C_{l}$ by a factor $\left(1+\alpha^{2}\right)$. We shall not investigate here this possibility but we can note that a significant effect would require rather large velocities.

\subsection{Free electron correlation function}

The angular correlation $C(\theta)$ and the power-spectrum $C_{l}$ correspond to the sum over the line of sight of the fluctuations of the free electron number density, up to the recombination redshift. Then, this integration over redshift could blur some features of these density fluctuations. Hence, in order to clarify the analysis it is interesting to consider first the real-space correlation function $\xi_{\mathrm{e}}(r ; z)$ obtained for a given redshift $z$. This also allows us to see the evolution with redshift of the free electron density fluctuations.

In order to understand the physical origin of the signal we split up the correlation function into several parts. First, we consider the total contribution $\xi_{\mathrm{e}}(r)$ from the IGM, as defined in (16). Next, we introduce $\xi_{\mathrm{e}, \mathrm{u}}$ as the 


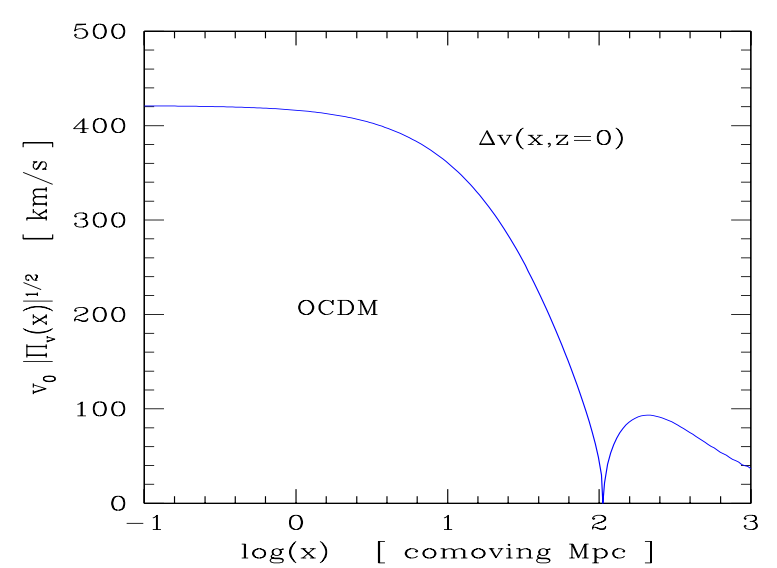

Fig. 1. The magnitude of the velocity correlation function $\xi_{\mathrm{v}}(x)$ at redshift $z=0$. The solid curve shows the quantity $v_{0}\left|\Pi_{\mathrm{v}}(x)\right|^{1 / 2}$, see (14). The feature at $x \simeq 100 \mathrm{Mpc}$ corresponds to the fact that $\Pi_{\mathrm{v}}(x)$ becomes negative for $x \geq$ $100 \mathrm{Mpc}$

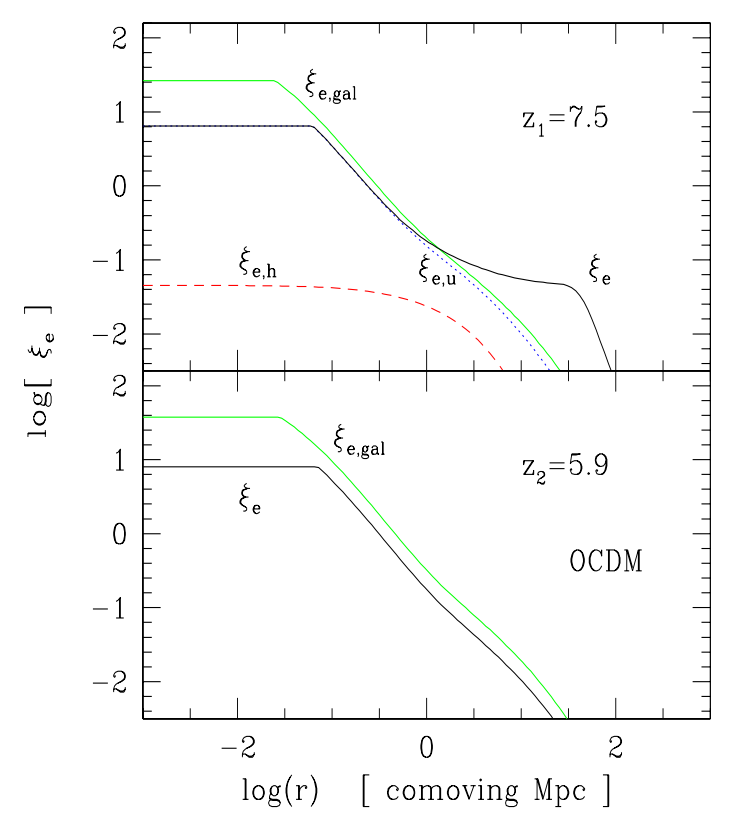

Fig. 2. The real-space two-point correlation function $\xi_{\mathrm{e}}(r)$ at two different redshifts. The solid line labeled $\xi_{\mathrm{e}}$ (resp. $\left.\xi_{\mathrm{e}, \text { gal }}\right)$ shows the contribution from the IGM (resp. from galactic halos). The curves $\xi_{\mathrm{e}, \mathrm{u}}$ and $\xi_{\mathrm{e}, \mathrm{h}}$ correspond to the "uncorrelated bubbles" and "homogeneous IGM" scenarios (see main text)

correlation function we get when we do not take into account the correlations of ionized bubbles (subscript " $\mathrm{u}$ " for "uncorrelated"). That is, in (20) we set the term $\xi_{x_{1}, x_{2}}(r)$ to 0 . Then, we define $\xi_{\mathrm{e}, \mathrm{h}}$ as the signal produced by uncorrelated bubbles into a "homogeneous" IGM (subscript "h" for "homogeneous"). That is, in (16) we set the term $\xi_{\mathrm{IGM}}(r)$ to 0 . Thus, $\xi_{\mathrm{e}, \mathrm{h}}$ allows us to see the contribution to $\xi_{\mathrm{e}}$ due to the inhomogeneities of the free electron number density produced by patchy reionization in distant bubbles. Then, $\xi_{\mathrm{e}, \mathrm{u}}$ shows by comparison with $\xi_{\mathrm{e}, \mathrm{h}}$ the importance of the clumping of the gas within the IGM. Finally, the difference between $\xi_{\mathrm{e}, \mathrm{u}}$ and the total signal $\xi_{\mathrm{e}}(r)$ measures the effect of the correlations of these ionized bubbles.
We show our results in Fig. 2 for two different redshifts. The upper panel at $z_{1}=7.5$ corresponds to a redshift slightly before reionization (at $z_{\mathrm{ri}}=6.8$ ). First, we clearly see on $\xi_{\mathrm{e}, \mathrm{h}}$ the contribution due to patchy reionization within finite size bubbles. Thus, $\xi_{\mathrm{e}, \mathrm{h}}$ is constant at small scales below the characteristic size of the ionized bubbles ( $\sim 0.5$ comoving $\mathrm{Mpc})$ and it drops at large scales. Of course, since $\xi_{\mathrm{e}, \mathrm{h}}$ only corresponds to fluctuations in the ionized fraction (it does not take into account matter density fluctuations) it is of the form $\xi_{\mathrm{e}, \mathrm{h}}(r)=$ $\left\langle x_{\mathrm{e}, 1} x_{\mathrm{e}, 2}\right\rangle(r)-\left\langle x_{\mathrm{e}}\right\rangle^{2}$. Since by definition we have $x_{\mathrm{e}} \leq 1$ we get $\xi_{\mathrm{e}, \mathrm{h}} \leq 1$. In fact, $\xi_{\mathrm{e}, \mathrm{h}}$ never reaches unity because at large redshift the filling factor $Q_{\mathrm{HII}}$ of the ionized bubbles is much smaller than unity while at low $z$ after reionization all the medium is reionized hence $\xi_{\mathrm{e}, \mathrm{h}}(r)=0$. In the upper panel we have $Q_{\mathrm{HII}} \simeq 0.9<1$ while in the lower panel after reionization we have $\xi_{\mathrm{e}, \mathrm{h}}(r)=0$ hence the curve does not appear in the figure.

Next, $\xi_{\mathrm{e}, \mathrm{u}}$ shows the influence of the clumping of the gas within the IGM. Since $C_{\mathrm{IGM}}>1$ we no longer have the upper bound $\xi_{\mathrm{e}, \mathrm{u}} \leq 1$ and we can check in the figure that indeed we can have $\xi_{\mathrm{e}, \mathrm{u}}>1$. However, the typical overdensities within the IGM are smaller than the density contrast $\Delta_{\mathrm{c}}(z)$ of just-virialized halos hence $C_{\mathrm{IGM}} \leq 1+\Delta_{\mathrm{c}}$, see the expression (27). Thus, we obtain $\xi_{\mathrm{e}, \mathrm{u}} \leq \Delta_{\mathrm{c}}(z)$. We can check in both panels in Fig. 2 that our results agree with this upper bound $\left(\Delta_{\mathrm{c}}(z) \sim 200\right)$. In fact, $\xi_{\mathrm{e}, \mathrm{u}}$ is significantly smaller $\left(\xi_{\mathrm{e}, \mathrm{u}} \lesssim 10\right)$ since most of the volume of the universe is filled by lower density regions with $\rho \lesssim \bar{\rho}$. In agreement with (29) the correlation function $\xi_{\mathrm{e}, \mathrm{u}}(r)$ saturates at small scales below the damping scale $R_{\mathrm{d}}$ and it follows the decrease at larger scales of the matter correlation function. Since $R_{\mathrm{d}}$ is smaller than the typical sizes of the ionized bubbles the characteristic break of $\xi_{\mathrm{e}, \mathrm{u}}(r)$ occurs at smaller scales than for $\xi_{\mathrm{e}, \mathrm{h}}$. In other words, the matter density fluctuations provide more small scale power in relative terms than ionized bubbles. Moreover, since the clumping of the gas is rather large, even at large redshifts (at $z \gtrsim 10$ we already have $C_{\text {IGM }} \gtrsim 10$, see Valageas \& Silk 1999a), we find that $\xi_{\mathrm{e}, \mathrm{u}} \gg \xi_{\mathrm{e}, \mathrm{h}}$ at all scales.

However, the presence of the ionized bubbles is not totally blurred by the superimposed matter density fluctuations. Indeed, we can see in the upper panel that at large scales the actual correlation $\xi_{\mathrm{e}}$ is much larger than $\xi_{\mathrm{e}, \mathrm{u}}$. This means that for $r \gtrsim 1$ comoving Mpc the signal is dominated by the cross-correlation of ionized bubbles. This arises from the correlations of their central collapsed halos (which are the sites of formation of the central radiation source, either a galaxy or a QSO), see (20), which correspond to very rare overdensities. Of course, by definition this effect appears at scales larger than the typical size of the ionized bubbles. It provides excess large scale power to $\xi_{\mathrm{e}}$, as compared with $\xi_{\mathrm{e}, \mathrm{u}}$, and it leads to a characteristic feature in the shape of the correlation function $\xi_{\mathrm{e}}(r)$. After reionization (lower panel) since there are no more ionized bubbles this effect disappears and $\xi_{\mathrm{e}}(r)$ is only governed by the fluctuations of the matter density, hence $\xi_{\mathrm{e}, \mathrm{u}}$ (not shown) becomes equal to $\xi_{\mathrm{e}}$. 


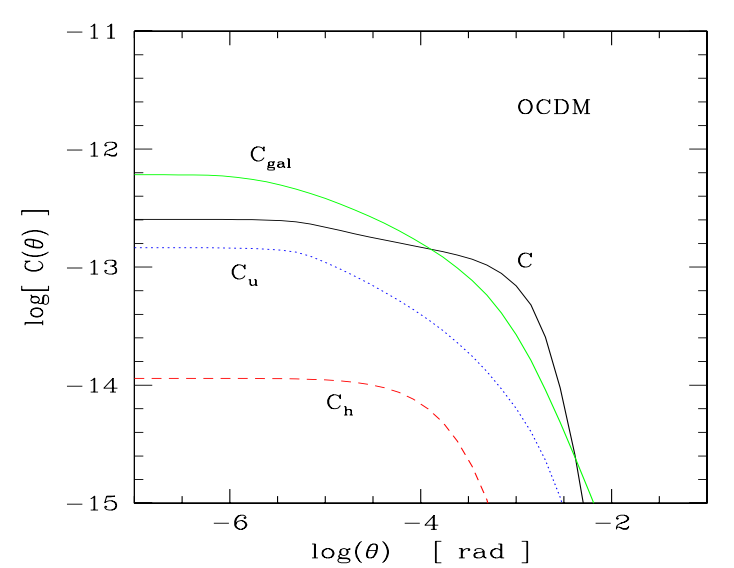

Fig. 3. The angular two-point correlation function $C(\theta)$. The solid line labeled $C$ shows the contribution from the IGM. The curve $C_{\text {gal }}$ displays the contribution from galactic halos. The curves $C_{\mathrm{u}}$ and $C_{\mathrm{h}}$ correspond to the "uncorrelated bubbles" and "homogeneous IGM" scenarios (see main text)

Finally, the curve $\xi_{\text {e,gal }}$ shows the contribution from galactic halos. We can see that the scale $R_{\text {cool }}$ is somewhat smaller than the damping scale $R_{\mathrm{d}}$ (this is related to the fact that the density of virialized halos is larger by a factor $\Delta_{\mathrm{c}} \sim 200$ than the typical IGM density) so that $\xi_{\mathrm{e}, \text { gal }}$ saturates at smaller scales than $\xi_{\mathrm{e}}$. Moreover, the clumping factor associated with these halos is larger than for the IGM (because of this difference between the typical densities) hence the plateau at small scales of $\xi_{\text {e,gal }}$ is higher than for $\xi_{\mathrm{e}}$. From (31) and (32) we can check that $\xi_{\text {e,gal }} \leq F_{\text {gal }} \Delta_{c} \leq \Delta_{c}$. We can see in the figure that $\xi_{\text {e,gal }}$ is somewhat smaller than this upper bound because the fraction of matter enclosed within such halos is still low at these redshifts: $F_{\text {gal }} \sim 0.12$ at $z \sim 7$ (see also Fig. 12 in Valageas \& Silk 1999a). We note that at larger scales $(r \sim 1 \mathrm{Mpc})$ the contribution from the IGM and "galaxies" are of the same order while at very large scales $(r>1 \mathrm{Mpc})$ before reionization the signal is dominated by the IGM through the cross-correlation of ionized bubbles. We can expect to recover these features in the integrated quantities $C(\theta)$ and $C_{l}$.

\subsection{Angular two-point correlation function}

We show in Fig. 3 our results for the angular two-point correlation function $C(\theta)$. We can check that we recover the trends described in Sect. 3.2, which have not been totally blurred by the integration over redshift.

First, we recover as in Fig. 2 the characteristic shape of the contribution $C_{\mathrm{h}}$ from pure ionized bubbles: a plateau at small angular scales $\theta \lesssim 10^{-4}$ rad and a sharp drop at larger scales, beyond the size of the bubbles. Note that a characteristic length scale $r$ is related to the angular scale $\theta$ by $r \sim \theta c / H_{0} \sim 5 \theta 10^{3}$ (in comoving Mpc). Hence $\theta \lesssim 10^{-4}$ corresponds indeed to the scale $r \sim 0.5 \mathrm{Mpc}$ seen in Fig. 2 (the knee of $\xi_{\mathrm{e}, \mathrm{h}}(r)$ ). Then, the correlation $C_{\mathrm{u}}$ shows a smoother shape, due to the power-law behaviour of the real-space matter correlation function $\xi(r)$, with a break at a smaller scale $\theta \sim 10^{-5.5} \mathrm{rad}$ due to small scale matter density fluctuations. Moreover, we have $C_{\mathrm{u}}>C_{\mathrm{h}}$, in agreement with Fig. 2. Next, the total signal $C(\theta)$ from the IGM is larger than $C_{\mathrm{u}}$, especially at large angular scales $\theta \sim 10^{-3} \mathrm{rad}$, because of the crosscorrelation of ionized bubbles. Note that the integration along the line of sight spreads the difference between $C_{\mathrm{u}}$ and $C$ over all angles $\theta$ (in particular down to $\theta \rightarrow 0$ ) since a small angular separation $\theta$ corresponds to a large realspace distance $r$ at high $z$. This leads to a difference with the real-space correlation $\xi_{\mathrm{e}}(r)$ shown in Fig. 2 where we found that at small scales (below the size of ionized bubbles) $\xi_{\mathrm{e}, \mathrm{u}}$ is equal to $\xi_{\mathrm{e}}$. In a similar fashion, the integration over redshift also leads to smoother curves $C(\theta)$. Finally, we can see that the contribution $C_{\text {gal }}$ from galactic halos is larger than the signal from the IGM at small scales $\theta \lesssim 10^{-4} \mathrm{rad}$ while it becomes smaller at larger scales, as expected from Fig. 2. However, on the whole the difference between both contributions is not very large.

\subsection{Power-spectra $C_{l}$ and $S_{I}$}

We show in Fig. 4 the quantity $l(l+1) C_{l} /(2 \pi)$ and the "local average" $S_{l}$, for the contributions from the IGM and from galactic halos. We also plot for comparison the power spectrum of primary anisotropies calculated using CMBFAST (Seljak \& Zaldarriaga 1996). At small $l$ both quantities $l(l+1) C_{l} /(2 \pi)$ and $S_{l}$ are almost identical since $C_{l}$ varies slowly with the wavenumber $l$. At large $l$ $\left(l \gtrsim 10^{5}\right)$ the power-spectrum $C_{l}$ exhibits an oscillatory behaviour (since it is the Fourier transform of a function $C(\theta)$ which shows a sharp drop at large angular scales) and a slow decline. In particular, at very large $l\left(l \gtrsim 10^{7}\right)$ the oscillations of $C_{l}$ are not resolved by the numerical calculation. On the other hand, $S_{l}$ becomes significantly different from $l(l+1) C_{l} /(2 \pi)$ as it shows a sharp decrease with $l$ and fewer oscillations. Of course, this is due to the "averaging procedure" which enters the definition (40) of $S_{l}$. At large $l$ the numerous oscillations of $C_{l}$ over the range $[l / 2,3 l / 2]$ partially cancel out which leads to a stronger falloff for $S_{l}$. Moreover, this "averaging" smoothes the behaviour of $S_{l}$ which shows much weaker oscillations. This also allows us to resolve $S_{l}$ up to larger $l$ (note that $S_{l}$ is not computed from $C_{l}$ but directly from the expression (41)). This suggests that for observational purposes too, the quantity $S_{l}$ may be more convenient as it should be more robust (i.e. require a lower resolution) than $C_{l}$ at large $l$ and it shows more clearly the transition to the large- $l$ regime by a sudden drop.

Note that for a correlation function $C(\theta)=$ $\exp \left[-\left(\theta / \theta_{0}\right)^{2}\right]$ with a Gaussian cutoff with a characteristic scale $\theta_{0}$ we get from (42):

$S_{l}=\mathrm{e}^{-\left(l \theta_{0}\right)^{2} / 16}-\mathrm{e}^{-9\left(l \theta_{0}\right)^{2} / 16} \sim \mathrm{e}^{-\left(l \theta_{0}\right)^{2} / 16}$

while (38) gives:

$\frac{l^{2} C_{l}}{2 \pi}=\frac{1}{2}\left(l \theta_{0}\right)^{2} \mathrm{e}^{-\left(l \theta_{0}\right)^{2} / 4}$. 


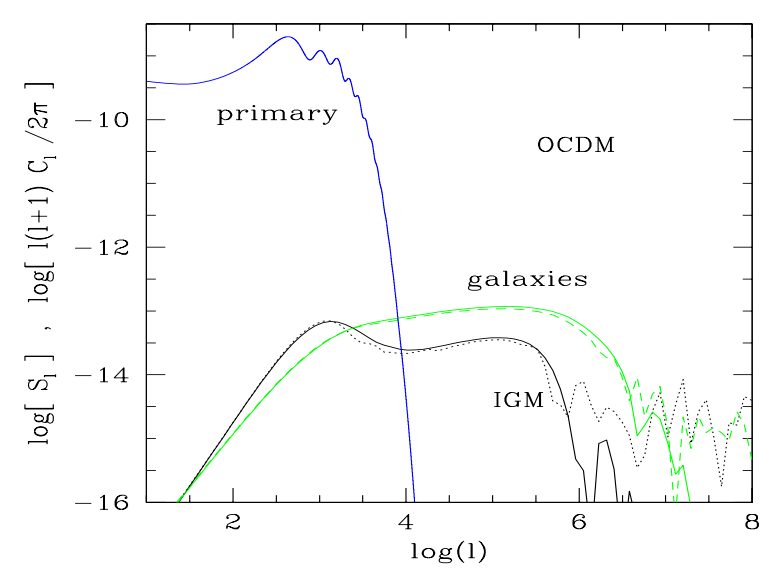

Fig. 4. The power-spectra $C_{l}$ and $S_{l}$ of the secondary anisotropies for the OCDM cosmology. The solid curves show the quantity $S_{l}$ for the contributions from the IGM and from galactic halos. The dotted (resp. dashed) curve with oscillations at large $l$ displays $l(l+1) C_{l} /(2 \pi)$ for the IGM (resp. galactic halos). The upper curve labeled "primary" shows $l(l+1) C_{l} /(2 \pi)$ of the primary anisotropies for the same OCDM model (see text)

On the other hand, for an exponential cutoff $C(\theta)=$ $\exp \left(-\theta / \theta_{0}\right)$ we obtain the same slow cutoff for both quantities: $S_{l} \sim l^{2} C_{l} \sim 1 /\left(l \theta_{0}\right)$. Finally, for a correlation function which is a top-hat (i.e. $C(\theta)=1$ for $\theta<\theta_{0}$ and $C(\theta)=0$ for $\left.\theta>\theta_{0}\right)$ we get:

$S_{l}=J_{0}\left(\frac{l \theta_{0}}{2}\right)-J_{0}\left(\frac{3 l \theta_{0}}{2}\right)$

and

$\frac{l^{2} C_{l}}{2 \pi}=\left(l \theta_{0}\right) J_{1}\left(l \theta_{0}\right)$.

In this latter case, at large $l$ both $C_{l}$ and $S_{l}$ show an oscillatory behaviour but we have $\left|S_{l}\right| \sim l^{-1 / 2}$ while $\left|l^{2} C_{l}\right| \sim l^{1 / 2}$, so that $S_{l}$ shows again a stronger decrease than $l(l+1) C_{l} /(2 \pi)$. Thus, these examples explicitly show that the shape of the large- $l$ tail of $S_{l}$ depends rather strongly on the details of the angular correlation function (and even more so for $C_{l}$ ). However, the "local average" $S_{l}$ usually shows a drop beyond the characteristic wavenumber $1 / \theta_{0}$ (while $\left|l^{2} C_{l}\right|$ may either decrease or grow in an oscillatory fashion).

The oscillatory behaviour of $C_{l}$ (hence the sharp drop of $S_{l}$ ) appears at lower $l$ for the IGM than for galactic halos. This is due to the fact that the correlations $\xi_{\mathrm{e}}(r)$ and $C(\theta)$ show less small-scale power for the IGM contribution than for the signal from galactic halos, as seen in Figs. 2 and 3. Indeed, as shown in (38) and (39) a characteristic angle $\theta$ (resp. a physical length $r$ ) translates into a characteristic wavenumber $l \sim 1 / \theta$ (resp. $\left.l \sim R_{z} / r\right)$. Hence, since the scales of the fluctuations of the free electron number density are larger for the IGM than for the galactic halo component the power-spectra $C_{l}$ and $S_{l}$ of the IGM appear shifted towards smaller $l$ with respect to the contribution from galaxies. At low $l$ we recover a white noise spectrum $\left(C_{l}\right.$ is constant, hence $\left.S_{l} \propto l^{2}\right)$ since this

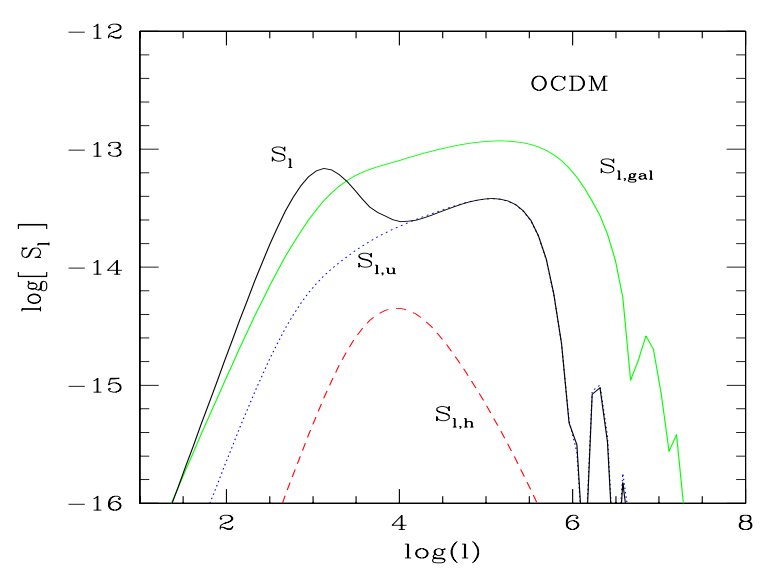

Fig. 5. The power-spectrum $S_{l}$ of the secondary anisotropies for the OCDM cosmology. The solid curve labeled $S_{l}$ (resp. $S_{l, \text { gal }}$ ) shows the contribution from the IGM (resp. from galactic halos). The curves $S_{l, \mathrm{u}}$ and $S_{l, \mathrm{~h}}$ correspond to the "uncorrelated bubbles" and "homogeneous IGM" scenarios

corresponds to very large scales where the correlations of the electron distribution are negligible. However, the slope of $S_{l}$ we find at low $l$ for the contribution from galactic halos is smaller because of the large-scale correlations of these rare overdense objects.

We note that we clearly recover the main features of the correlation $C(\theta)$ shown in Fig. 3. Thus, for the IGM we find that the transition to the white noise part (the falloff at low $l$ with a slope $l^{2}$ ) occurs at $l \sim 10^{3}$ which corresponds to the cutoff at large angles $\theta \sim 10^{-3} \mathrm{rad}$ of the correlation $C(\theta)$ (see the strong knee in Fig. 3). On the other hand, for the galactic halo contribution the transition to the large- $l$ regime (marked by the drop of $S_{l}$ ) appears at $l \sim 10^{6}$ which corresponds to the scale $\theta \sim 10^{-6}$ rad below which $C_{\text {gal }}(\theta)$ saturates (hence to the smallest angular scale of the density fluctuations). Note that additional power at smaller scales (due to substructures within halos and to the subsequent collapse of baryons when they cool) would shift this transition towards higher $l$.

In a fashion similar to Fig. 3 we can split up the spectrum $S_{l}$ into several components. This decomposition is shown in Fig. 5. First, in agreement with Figs. 2 and 3 we can see that the contribution $S_{l, \mathrm{~h}}$ from uncorrelated ionized bubbles within a uniform medium is strongly peaked at $l \sim 10^{4}$ which corresponds to the typical size of the ionized bubbles $\left(\theta_{0} \sim 10^{-4} \mathrm{rad}, r \sim 0.5\right.$ comoving $\left.\mathrm{Mpc}\right)$. At small wavenumber $\left(l \theta_{0}\right) \ll 1$ we recover a white noise spectrum $S_{l} \sim\left(l \theta_{0}\right)^{2}$ while at larger $l$ we get a somewhat smoother decrease than $l^{-2}$.

Next, in agreement with Fig. 3 we can see that $S_{l, \mathrm{u}}$ is larger than $S_{l, \mathrm{~h}}$ and it shows a broader maximum, due to the additional power at small and large scales provided by the density fluctuations within the IGM. The comparison with the total signal $S_{l}$ shows that this Fourier transform actually separates the various contributions to the power-spectrum. Thus, for $l>10^{4}$ we have $S_{l}=S_{l, \mathrm{u}}$ and the peak at $l \sim 10^{5.5}$ corresponds to the break at $\theta \sim 10^{-5.5}$ of the correlation $C_{\mathrm{u}}(\theta)$ (and to the 
saturation at $r \sim 210^{-2} \mathrm{Mpc}$ of $\left.\xi_{\mathrm{e}, \mathrm{u}}(r)\right)$. This directly probes the small-scale fluctuations of the matter density field. Moreover, the equality $S_{l}=S_{l, \mathrm{u}}$ in this large- $l$ regime translates the fact that at small scales $r<1 \mathrm{Mpc}$ we had $\xi_{\mathrm{e}}=\xi_{\mathrm{e}, \mathrm{u}}$, as seen in Fig. 2. Note that in the angular space representation $C(\theta)$ the integration over redshift along the line of sight destroys this feature as $C(\theta)>C_{\mathrm{u}}(\theta)$ for all angles and one cannot recognize from the total signal $C$ the signature of this small scale feature. Thus, the Fourier transform presents the strong advantage to separate various physical processes as they act on different scales. However, in this regime $l>10^{4}$ the signal should be dominated by the contribution from galactic halos.

Here we can note that in our calculations we modelled ionized regions as spherical bubbles while detailed numerical simulations show they can display a more complex morphology (e.g., Abel et al. 1999) as ionization fronts propagate more easily in voids. This means that the bellshaped curve $S_{l, \mathrm{~h}}$ in Fig. 5 (which measures the contribution from patchy reionization, i.e. from the geometry of HII regions) underestimates the actual signal at large wavenumbers $\left(l>10^{4}\right)$ where we neglected the contribution from higher-order spherical harmonics (which provide some power on scales smaller than the typical radius $R_{\mathrm{i}}$ of the ionized bubble). However, we can check in Fig. 5 that even if we spread the maximum of the curve $S_{l, \mathrm{~h}}$ up to wavenumbers $l$ ten times larger (i.e. the geometry of the ionization front displays significant power up to scales ten times smaller than $R_{\mathrm{i}}$ ) our results remain unchanged. Indeed, most of the power in this range is due to the fluctuations of the matter density itself rather than to the geometry of HII regions. Moreover, the smallest scales displayed by the geometry of the ionization fronts are at least of the same order as the size of the smallest structures of the density field (from which they originate). Then, the factor $n_{\mathrm{e}}^{2}$ in the correlation $C(\theta)$ ensures that if a large fraction of such clouds, enclosed within the distance $R_{\mathrm{i}}$ to the radiation source, are ionized and if they show a density contrast $\delta \gtrsim 10$, the signal is dominated on these scales by the fluctuations of the free electron density rather than by sheer geometrical patterns. Hence our results are not very sensitive to the approximation of spherical HII bubbles.

Then, the comparison of $S_{l}$ with $S_{l, \mathrm{u}}$ shows that the peak at $l \sim 10^{3}$ comes from the correlations of ionized bubbles. This also corresponds to the knee at $\theta \sim 10^{-3} \mathrm{rad}$ of $C(\theta)$. In agreement with Fig. 3 we find that at small wavenumbers $l \lesssim 10^{3}$ the power-spectrum is dominated by the IGM contribution (i.e. the correlations of ionized bubbles) while at larger $l$ most of the signal comes from galactic halos which provide much small scale power. The peak at $l \sim 10^{6}$ of $S_{l, \text { gal }}$ corresponds to the break at $\theta \sim$ $10^{-6} \mathrm{rad}$ of $C_{\text {gal }}$. Thus, we see that the power-spectrum of the final signal mainly probes the small-scale density fluctuations which give rise to galactic halos (and their possible substructures) and the correlations at large scales of ionized bubbles.
Analytical studies of the power-spectrum $C_{l}$ of these secondary CMB anisotropies have also been presented in some earlier works. Thus, Gruzinov \& Hu (1998) consider the effect of patchy reionization within a uniform medium, assuming a Gaussian falloff for the real-space correlation function $\xi_{\mathrm{e}}(r)$. This could arise from a Gaussian distribution of the size of the HII regions. Then, as in (43) and (44) they obtain a Gaussian cutoff for the angular correlation $C(\theta)$ and the power-spectra $S_{l}$ and $C_{l}$. This corresponds to our curves $C_{\mathrm{h}}$ and $S_{l, \mathrm{~h}}$ in Figs. 3 and 5. Our predictions for this scenario are similar to their results, but our spectrum $S_{l}$ peaks at a larger $l\left(l \sim 10^{4}\right)$ than theirs $\left(l \sim 10^{3}\right)$. This is due to the fact that in our model the comoving size of HII regions is of order $0.5 \mathrm{Mpc}$ (see Fig. 2) while they assume a very large radius of $20 \mathrm{Mpc}$ for the bubbles. We can also note that our cutoff at large $l$ is smoother than a Gaussian. Indeed, since observed luminosity functions usually show a simple exponential cutoff (and our results match observations at low $z$ ) we can expect a shallow cutoff of the form $\exp \left(-r^{1 / 3}\right)$ (because the volume of ionized bubbles is proportional to the number of ionized atoms, hence to the luminosity of the source) and we noticed above that a pure exponential cutoff already leads to a simple power-law decline of the spectrum $S_{l}$ (as $\left.1 / l\right)$. Using a slightly more sophisticated model, Aghanim et al. (1996) calculate the reionization from early formed quasars, deducing the statistic of the ionized bubbles size from the distribution of quasar luminosities. Their results predict that most of the power is at $l \sim 10^{3}$ and they are similar to a one-patch scenario with a bubble radius of $\sim 10 \mathrm{Mpc}$, except for the high $l$ cutoff which is less steep, due to the smaller patches distribution. Then, Knox et al. (1998) consider the effect of the correlations of these ionized bubbles. In agreement with our results, they find that this leads to a much broader distribution of the power-spectrum. Note that in our analysis we have split up the influence of matter correlations into two processes: the cross-correlation of HII regions themselves (through the correlation of the emitting sources), which provides additional power at larger scales $\left(l<10^{4}\right)$ than the size of these patches, and the fluctuations of the matter density field within these bubbles, which builds power at smaller scales $\left(l>10^{4}\right)$. Thus, the final signals $C(\theta)$ and $S_{l}$ are the sum of the contributions from various processes. The advantage of our approach is that it provides a fully consistent description of these different scales, from a model built to study the detailed reionization history of the universe which has already been compared with observations for various aspects (e.g., galaxy luminosity function in Valageas \& Schaeffer 1999; X-ray emission from clusters, galaxies and quasars in Valageas \& Schaeffer 2000).

\subsection{Redshift distribution}

The angular correlation $C(\theta)$ and the power-spectra $C_{l}$ and $S_{l}$ correspond to an integration along the line of 


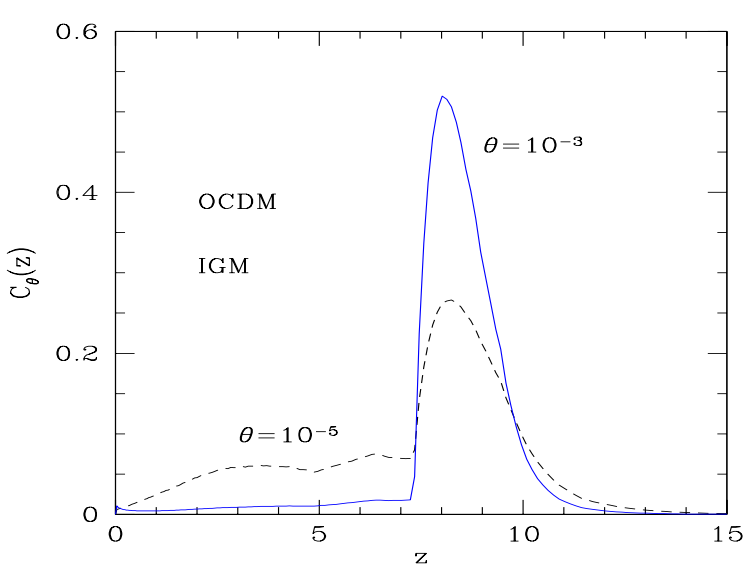

Fig. 6. The redshift distribution $C_{\theta}(z)$ of the angular correlation (normalized to unity) from the IGM, for $\theta=10^{-3} \mathrm{rad}$ (solid line) and $\theta=10^{-5} \mathrm{rad}$ (dashed line)

sight of the fluctuations of the free electron number density. However, it would be interesting to see the relative importance of the contributions from various redshifts to the final signal. In particular, this would show whether these secondary CMB anisotropies arise from a narrow range of redshifts close to reionization at $z_{\mathrm{ri}}=6.8$ or from a more extended interval. Thus, we define the normalized quantity $C_{\theta}(z)$ by:

$C_{\theta}(z)=\frac{2 \tau_{0}^{2} v_{0}^{2}}{3 c^{2} C(\theta)} w(z) \int_{R_{\theta}}^{\infty} \frac{\mathrm{d} r}{R_{z}} \frac{r \Pi_{\mathrm{v}}[r(1+z)] \xi_{\mathrm{e}}(r)}{\sqrt{r^{2}-R_{\theta}^{2}}}$.

It obeys the normalization condition:

$\int_{0}^{\infty} \mathrm{d} z C_{\theta}(z)=1$

and $C_{\theta}(z) \mathrm{d} z$ is the fraction of the final angular correlation function which is generated in the redshift interval $\mathrm{d} z$. In a similar fashion we define the quantities $C_{l}(z)$ and $S_{l}(z)$, from (39) and (41), which are also normalized to unity. For these three redshift distributions we alternatively consider the contributions from the IGM and from galactic halos.

\subsubsection{IGM contribution}

First, we consider the contributions from the IGM to the secondary CMB anisotropies. We show our results for the redshift distribution $C_{\theta}(z)$ of the angular correlation in Fig. 6, for two different angular scales. We note that the contributions to the final signal $C(\theta)$ come from a rather large range of redshifts, typically $7.5<z<10$ (so that $\left.\delta z / z_{\mathrm{ri}} \sim 0.36\right)$. There is a sharp cutoff at $z \simeq z_{\mathrm{ri}}$ since at lower redshifts there are no more ionized bubbles. This drop is sharper for larger angular scales, in agreement with Figs. 3 and 5 where we noticed that large scales $\theta>10^{-4} \mathrm{rad}\left(l<10^{4}\right)$ are dominated by the correlations of ionized bubbles. However, at small scales $\theta \lesssim 10^{-5}$ there is a non-negligible tail at lower redshifts due to the matter density fluctuations within the fully ionized IGM. Of course, smaller scales also show a slightly more extended tail at high $z$ since at higher redshift the typical size of

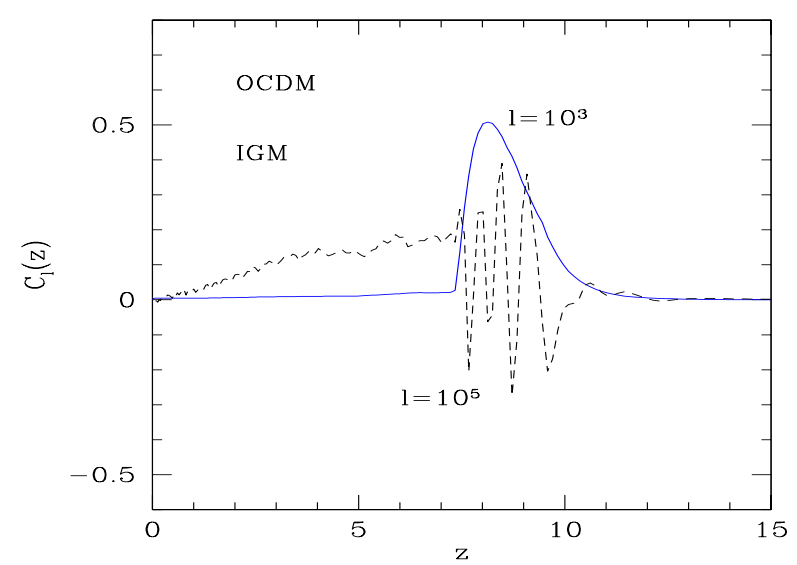

Fig. 7. The redshift distribution $C_{l}(z)$ of the power-spectrum $C_{l}$ (normalized to unity) from the IGM, for $l=10^{3}$ (solid line) and $l=10^{5}$ (dashed line)

ionized bubbles and the correlation length of the matter density field were smaller, which damps the contribution to large angular scales $\theta$. Hence the redshift distribution of the angular correlation $C(\theta)$ is somewhat broader for lower $\theta$ (which translates into the smaller height of the maximum of $C_{\theta}(z)$ in the figure since the curves are normalized to unity).

We display in Fig. 7 the redshift distribution $C_{l}(z)$ of the contribution from the IGM to the power-spectrum $C_{l}$ for two different wavenumbers (normalized to unity). Of course, for $l=10^{3}$ we recover a shape similar to the redshift distribution $C_{\theta}(z)$ we obtained for $\theta=10^{-3} \mathrm{rad}$, since both quantities correspond to the same scale. For $l=10^{5}$ the envelope of $C_{l}$ agrees again with the shape we got in Fig. 6 for $C_{\theta}(z)$ (with a tail at low $z$ due to small-scale density fluctuations) but the distribution $C_{l}(z)$ now shows several oscillations. This is due to the Fourier transform involved in the definition of $C_{l}$. Thus, the contributions from successive redshifts along the line of sight almost cancel out. This agrees with the behaviour we obtained in Fig. 4. Note that the oscillations occur before reionization: they are due to the patchy pattern of reionization in HII bubbles with a size larger than $R_{z} / l$. At lower redshift this feature disappears as there are no more ionized regions to single out a large characteristic scale.

Finally, we display in Fig. 8 the redshift distribution $S_{l}(z)$ of the contribution from the IGM to the powerspectrum $S_{l}$. As expected, for $l=10^{3}$ we recover the results we obtained for the power-spectrum $C_{l}$. Indeed, as noticed in Fig. 4 for small wavenumbers there are no oscillations since one probes scales which are larger or of the order of the correlation lengths of the free electron distribution, so that $S_{l} \simeq l(l+1) C_{l} /(2 \pi)$. At larger $l$ some oscillations start to appear and $S_{l}(z)$ shows a different shape than $C_{l}(z)$. In particular, the oscillations of $S_{l}(z)$ are much smoother and broader than for $C_{l}(z)$ and they appear at a larger wavenumber. Indeed, the "local averaging" over $l$ associated with the procedure used to define $S_{l}$ "smoothes" the contributions from various scales. 


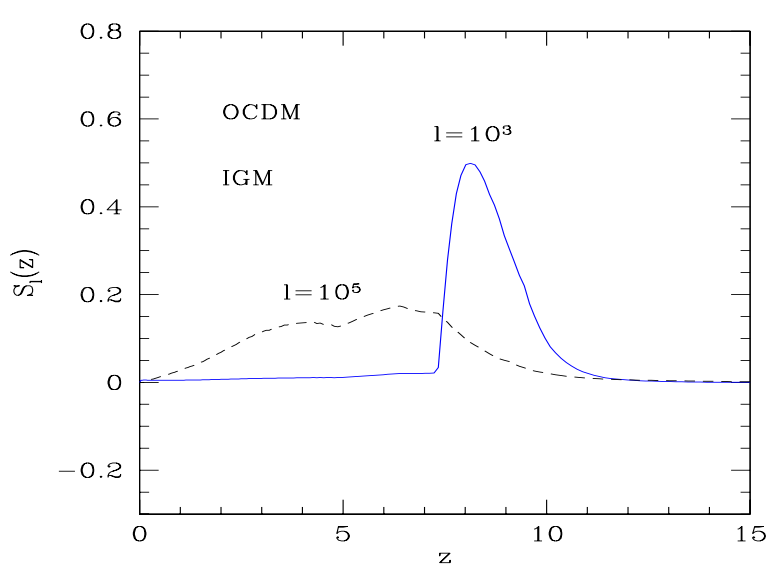

Fig. 8. The redshift distribution $S_{l}(z)$ of the power-spectrum $S_{l}$ (normalized to unity) from the IGM, for $l=10^{3}$ (solid line) and $l=10^{5}$ (dashed line)

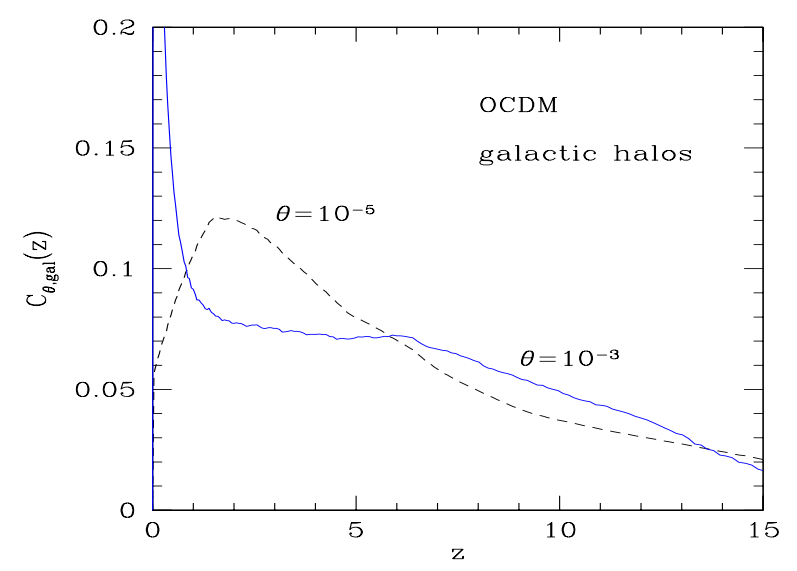

Fig. 9. The redshift distribution $C_{\theta, \text { gal }}(z)$ of the angular correlation (normalized to unity) from galactic halos, for $\theta=$ $10^{-3} \mathrm{rad}$ (solid line) and $\theta=10^{-5} \mathrm{rad}$ (dashed line)

In particular, this allows us to see more clearly the redshift distribution associated with $l=10^{5}$ where there are no oscillations yet. Moreover, it clearly shows that the large oscillations we obtained shortly before reionization for $C_{l}$ almost cancel out so that high redshifts $z>z_{\mathrm{ri}}$ only provide a small contribution to the final signal. This leads to a redshift distribution which is very different from the one obtained for smaller $l$ which shows a sharp cutoff at $z \simeq z_{\text {ri }}$. Thus, we find that for these small scales the contributions to the power-spectrum $S_{l}$ come from an extended range of redshifts $2<z<8$. As noticed in Fig. 5, we find again that the use of the power-spectrum allows one to clearly see the various processes associated with different scales, which are somewhat blurred in the angular representation $C(\theta)$.

\subsubsection{Contribution from galactic halos}

Now, we consider the redshift distribution of the contribution from galactic halos to the CMB anisotropies. We show in Fig. 9 our results for the angular correlation function. First, we note that there is no drop at the reionization redshift $z_{\mathrm{ri}}$. Indeed, the fact that ionized bubbles

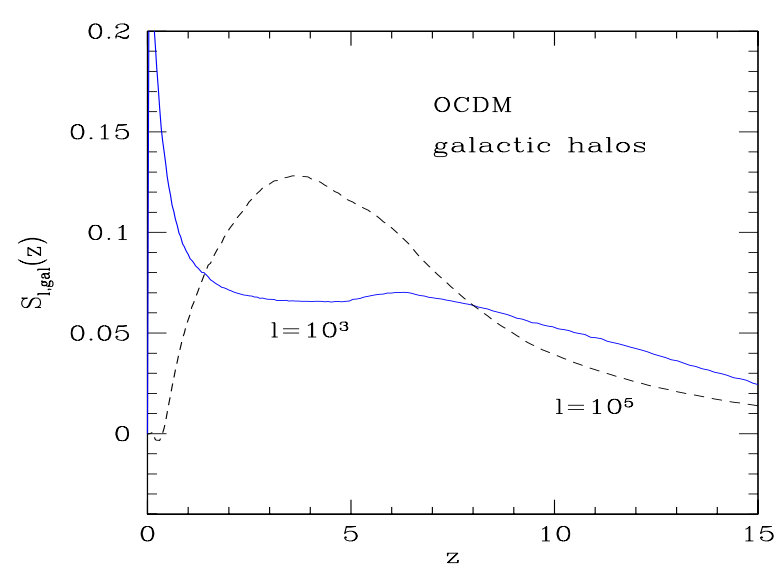

Fig. 10. The redshift distribution $S_{l, \text { gal }}(z)$ of the powerspectrum $S_{l}$ (normalized to unity) from galactic halos, for $l=10^{3}$ (solid line) and $l=10^{5}$ (dashed line)

suddenly overlap so that the signal from the patchy pattern of reionization disappears does not affect the contribution from galactic halos. On the other hand, reionization does not lead to a sharp drop of the galaxy or quasar multiplicity functions either since it does not imply a sudden increase of the IGM temperature and of the Jeans mass. Indeed, as seen in Valageas \& Silk (1999a) most of the reheating of the universe occured earlier in a gradual fashion so that the small increase of the IGM temperature at $z_{\text {ri }}$ has no impact on the population of radiation sources. Thus, the redshift distribution $C_{\theta, \text { gal }}(z)$ follows the growth of non-linear structures so that smaller redshifts provide a larger contribution. This appears clearly for $\theta=$ $10^{-3} \mathrm{rad}$ where most of the signal is generated at $z \lesssim 1$ when the scale $r \sim 5 \mathrm{Mpc}$ enters the non-linear regime. On the other hand, for the smaller angular scale $\theta=$ $10^{-5}$ rad the contribution from very low $z$ becomes smaller as the typical size of virialized objects becomes larger than the scale which corresponds to $\theta$. However, we may underestimate the power at low $z$ because we neglected substructures within halos. Note that on these small angular scales the secondary CMB anisotropies should be dominated by the contribution from galactic halos. Hence they arise from a very broad range of redshifts (typically $0<z<7)$ which is not related to $z_{\mathrm{ri}}$.

We display in Fig. 10 the redshift distribution $S_{l, \text { gal }}(z)$ of the power-spectrum $S_{l, \text { gal }}$. We recover a behaviour similar to Fig. 9. In particular, note the large range of redshifts which is probed by large wavenumbers $l \sim 10^{5}$. Higher $l$ which are beyond the cutoff of the spectrum $S_{l}$ show increasingly important oscillations.

\subsection{Quasars versus stars}

In usual scenarios the universe is reionized by the radiation emitted by non-linear structures as collisional ionization is likely to be less efficient (e.g., Madau 2000; Valageas \& Silk 1999b). There are two natural sources of radiation in present cosmological models: stars and quasars. In particular, in our model the universe is reionized when 


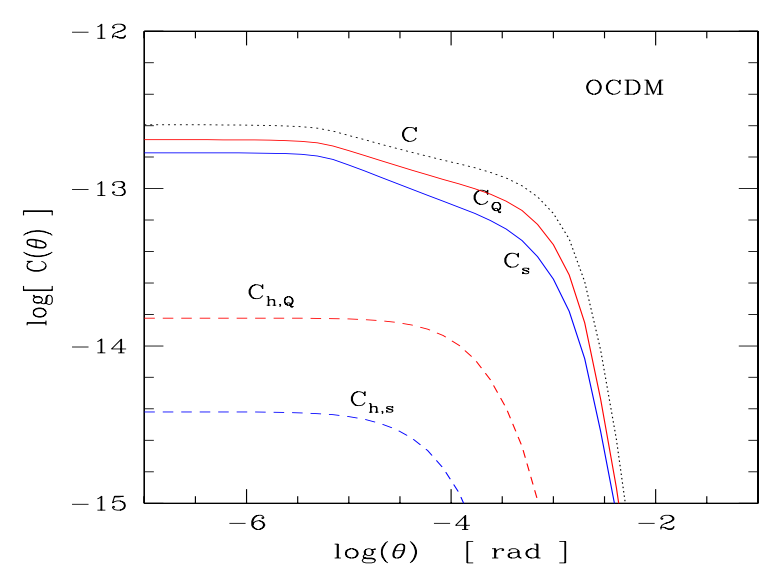

Fig. 11. The angular two-point correlation functions $C_{\mathrm{s}}(\theta)$ and $C_{\mathrm{Q}}(\theta)$ due to stars and quasars. The solid line labeled $C_{\mathrm{s}}$ (resp. $C_{\mathrm{Q}}$ ) shows the contribution from the IGM when we only take into account the ionized bubbles created by a central QSO (resp. by a central galaxy). The curves $C_{\mathrm{h}, \mathrm{s}}$ (resp. $C_{\mathrm{h}, \mathrm{Q}}$ ) corresponds to the "homogeneous IGM" scenario of ionized bubbles created by stellar radiation (resp. quasars) within a uniform medium. The dotted line shows the total IGM correlation function $C(\theta)$ (it is identical to the solid line $C$ in Fig. 3)

HII bubbles created by galaxies and quasars overlap at $z_{\text {ri }}$ (see Valageas \& Silk 1999a). The multiplicity functions we use for galaxies and QSOs are normalized to the lowredshift universe $(z<4)$ and are obtained in a consistent fashion (see also Valageas \& Schaeffer 1999). Then, we find that the energy output provided by QSOs is of the same order as the energy radiated by stars. However, the spatial features of these two reionization processes may be different since one can expect QSOs to create fewer but more extended HII bubbles, since quasars are not as numerous as galaxies but their luminosity is much larger. Hence, the correlation function $C(\theta)$ and the power-spectrum $C_{l}$ may show more large-scale power for a quasar-driven reionization than for a galaxy-driven process. This is quite interesting as it might allow one to discriminate both scenarios. Note on the other hand that the reionization of helium is usually due to the radiation emitted by quasars, as in our model. Indeed, stars have a black-body spectrum which yields very few high energy photons while quasars exhibit a harder power-law spectrum over the relevant frequency range. However, as pointed out by Tumlinson \& Shull (2000) population III metal-free stars have a harder spectrum than typical low- $z$ stars, hence they might be able to ionize helium in addition to hydrogen. Thus, the relative importance of quasars and stars is still an open problem. Unfortunately, we shall see below that the observation of the secondary anisotropies of the CMB is unlikely to answer this problem.

Thus, we define $C_{\mathrm{s}}$ and $C_{\mathrm{h}, \mathrm{s}}$ (resp. $C_{\mathrm{Q}}$ and $\left.C_{\mathrm{h}, \mathrm{Q}}\right)$ as the angular correlation functions we obtain for the total IGM signal and for the contribution due to patchy reionization through uncorrelated ionized bubbles within a uniform IGM when we only count in our model the bubbles created by stellar radiation (resp. quasar radiation). In other words, we use the reionization history described

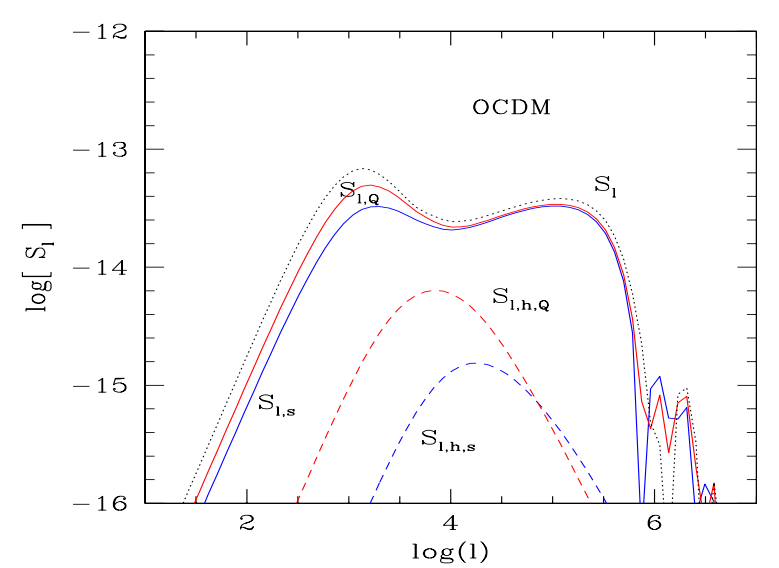

Fig. 12. The power-spectra $S_{l, \mathrm{~s}}$ and $S_{l, \mathrm{Q}}$ due to stars and quasars. The dashed curve $S_{l, \mathrm{~h}, \mathrm{~s}}$ (resp. $S_{l, \mathrm{~h}, \mathrm{Q}}$ ) corresponds to the "homogeneous IGM" scenario for ionized bubbles created by stellar radiation (resp. quasars) within a uniform medium. The dotted line shows the total IGM spectrum $S_{l}$ (it is identical to the solid line $S_{l}$ in Fig. 5)

in the previous sections (see Valageas \& Silk 1999a) but to compute the CMB secondary anisotropies we only take into account the bubbles associated with either one of the two available sources of radiation (stars or quasars). This allows us to compare the importance of stars and QSOs in our results (for the peculiar scenario of structure formation we use). We show our results in Fig. 11. First, we note that we recover for both cases the main features described in Sect. 3.3 for the total signal. Then, as expected, the comparison of $C_{\mathrm{h}, \mathrm{s}}$ with $C_{\mathrm{h}, \mathrm{Q}}$ shows that the characteristic scale of the ionized bubbles associated with quasars is larger than for the HII regions produced by galaxies. However, the difference is not very large (note that the radius only scales as $L^{1 / 3}$, where $L$ is the source luminosity, and one has to integrate over an extended population of sources and over redshift). Moreover, we find that the total signals $C_{\mathrm{s}}$ and $C_{\mathrm{Q}}$ are very close and they do not exhibit different characteristic scales. Indeed, as we described in Sect. 3.3 most of the power is provided by the small-scale matter density fluctuations of the IGM and by the large-scale cross correlations of ionized bubbles. Hence the typical size of the ionized bubbles cannot be seen in the shape of the angular correlation $C(\theta)$. Besides, since quasars and galaxies are drawn from the same population of collapsed halos they have similar correlations hence the cross-correlations of their associated HII regions are rather close (see Valageas et al. 2000 for a detailed study of the correlation properties of these various objects).

We show in Fig. 12 the power-spectra $S_{l, \mathrm{~s}}$ and $S_{l, \mathrm{Q}}$ (as well as $S_{l, \mathrm{~h}, \mathrm{~s}}$ and $S_{l, \mathrm{~h}, \mathrm{Q}}$ for the "homogeneous" scenario) associated with stars and quasars, which also correspond to the correlations displayed in Fig. 11. We find again that the spectra $S_{l, \mathrm{~h}, \mathrm{~s}}$ and $S_{l, \mathrm{~h}, \mathrm{Q}}$ which directly probe the size of the HII regions exhibit two slightly different scales for quasars and stars, in agreement with Fig. 11. Thus, $S_{l, \mathrm{~h}, \mathrm{~s}}$ peaks at $l \sim 10^{4.3}$ while $S_{l, \mathrm{~h}, \mathrm{Q}}$ peaks at $l \sim 10^{3.8}$. The wavenumber associated with quasar-driven bubbles is 
smaller than for stellar radiation since the size of the HII region is larger. However, we find again that this signature is lost in the total power-spectra $S_{l, \mathrm{~s}}$ and $S_{l, \mathrm{Q}}$ which are dominated at all scales by other processes (i.e. the correlations of the matter density field itself). Thus, observations of the secondary anisotropies of the CMB are unlikely to provide strong constraints on the size of the ionized bubbles. Hence they cannot discriminate between both sources of radiation (stars versus quasars).

Of course, this conclusion relies on the assumption that quasars are closely associated with galaxies. More precisely, our model is based on the usual scenario where QSOs correspond to massive black holes located in the nuclei of galaxies and powered by accretion (e.g., Rees 1849). Thus, an "exotic" model where quasars would not reside within massive virialized halos similar to galaxies might provide a different signature on the CMB. However, such a scenario is rather unlikely (e.g., in view of the energy requirements to power the quasars which favor large gravitational potential wells) and the standard model has been shown to agree reasonably well with numerous observations (e.g., the $B$-band luminosity functions and the X-ray emission, Valageas \& Schaeffer 2000). As we have shown above, on small scales $\left(l>10^{4}\right)$ the signal comes from the fluctuations of the density field within the IGM and from galactic halos, while the pattern of reionization plays a minor role. Hence our results in this range do not strongly depend on the clustering properties of quasars. On the other hand, on larger scales the kinetic SZ effect probes the spatial correlations of QSOs and in this sense it becomes more "model-dependent". However, we can be reasonably confident in our results as our model has already been checked against observations of the QSO multiplicity functions (e.g., Valageas \& Schaeffer 2000). Moreover, as shown in Fig. 8 in Valageas et al. (2000) we also recover the observed behaviour with redshift of the correlation length associated with quasars. This means that any model which satisfies the same observational constraints (up to $z \lesssim 5$ ) is likely to give analoguous results. Note that although the clustering properties of QSOs and galaxies as a whole are similar, since they are drawn from similar collapsed halos, the observed redshift-dependence of their correlation length is qualitatively different if one selects objects by a given luminosity threshold, due to the different behaviour of their mass-luminosity relations (see Valageas et al. 2000 for a detailed discussion).

\subsection{Dependence on cosmology}

Finally, in order to check whether our results strongly depend on the reionization history of the universe we also study the case of a standard CDM cosmology (SCDM): $\Omega_{\mathrm{m}}=1$. We use the same model as in Valageas \& Silk (1999a): $\Omega_{\mathrm{b}}=0.04, H_{0}=60 \mathrm{~km} \mathrm{~s}^{-1} / \mathrm{Mpc}$ and $\sigma_{8}=0.5$. The physical processes included in the model are the same as for the open cosmology and the galaxy and quasar multiplicity functions are again normalized to observations at

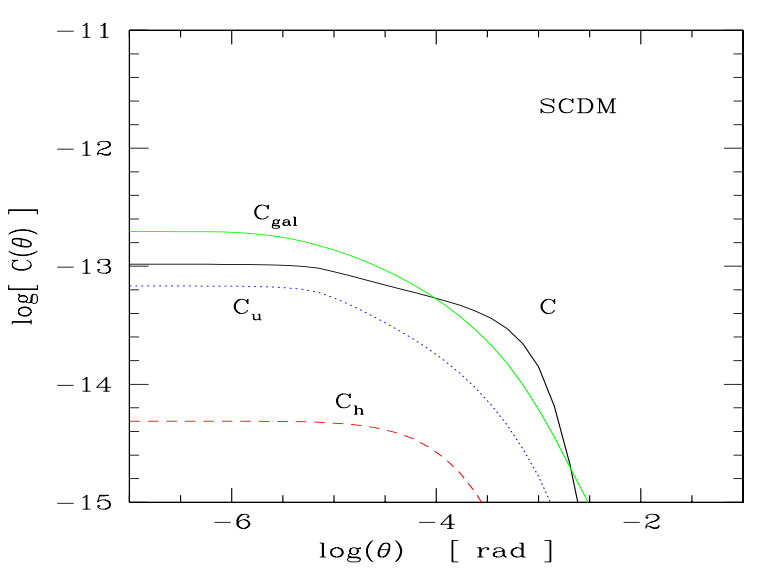

Fig. 13. The angular two-point correlation function $C(\theta)$ for a SCDM cosmology. The solid line labeled $C$ shows the contribution from the IGM. The curve $C_{\text {gal }}$ displays the contribution from galactic halos. The curves $C_{\mathrm{u}}$ and $C_{\mathrm{h}}$ correspond to the "uncorrelated bubbles" and "homogeneous IGM" scenarios, as in Fig. 3

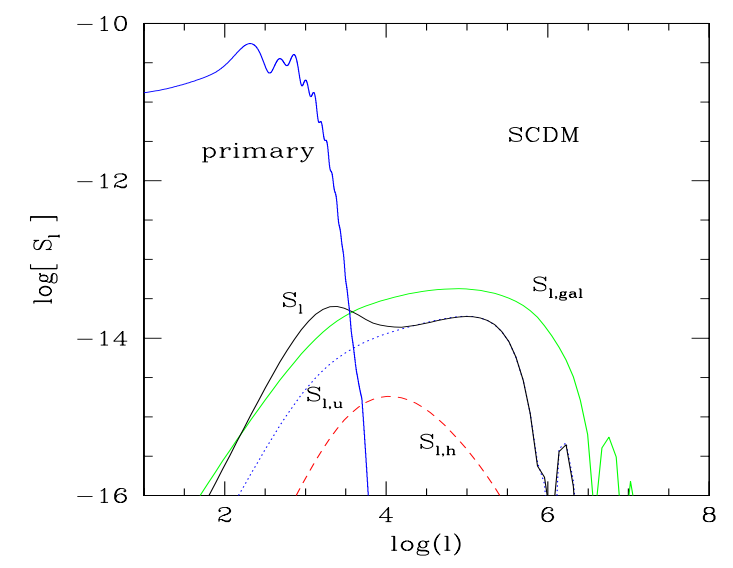

Fig. 14. The power-spectrum $S_{l}$ of the secondary anisotropies for the SCDM cosmology. The solid curve labeled $S_{l}$ (resp. $S_{l, \text { gal }}$ ) shows the contribution from the IGM (resp. from galactic halos). The curves $S_{l, \mathrm{u}}$ and $S_{l, \mathrm{~h}}$ correspond to the "uncorrelated bubbles" and "homogeneous IGM" scenarios, as in Fig. 5. The upper curve labeled "primary" shows $l(l+1) C_{l} /(2 \pi)$ of the primary anisotropies for the same SCDM model (see text)

low $z$ (Valageas \& Schaeffer 1999; Valageas \& Silk 1999a). The reionization redshift we get in this scenario is lower than previously: we now have $z_{\mathrm{ri}}=5.6$.

We show in Figs. 13 and 14 the angular correlation $C(\theta)$ and the power-spectrum $S_{l}$ we obtain for this critical density universe. First, we note that the amplitude of the secondary distortions of the CMB is smaller than for the previous cosmology. This is due to the smaller reionization redshift $z_{\mathrm{ri}}$. Indeed, the expression (34) shows that:

$C(\theta) \sim \delta z w\left(z_{\mathrm{ri}}\right) \sim \delta z\left(1+z_{\mathrm{ri}}\right)^{3 / 2}$

where $\delta z$ is the redshift interval during which reionization occurs. The smaller reionization redshift is due to the smaller variance $\sigma_{8}$ and to the faster growth with redshift of density fluctuations (which implies that for the same normalization at $z=0$ structure formation was less advanced at high redshift for the SCDM cosmology). Next, 
we can check in the figures that we recover the same features for the secondary anisotropies as for the open universe. In particular, we obtain the same shapes for all contributions to the correlation functions and the relative importance of each process remains unchanged. Hence all the points we discussed in the previous sections for the low-density cosmology remain valid. This is not surprising since the basic astrophysical model we use is the same. However, it shows that our conclusions do not strongly depend on the details of the model (e.g., the reionization redshift).

For these reasons, we expect that for low-density flat models, such as the "cosmic concordance" $\Lambda$ CDM model (Ostriker \& Steinhardt 1995; Krauss \& Turner 1995), the results should be similar to the OCDM model, except for a shift of the features to lower $l$ (larger angular scales) due to the different angular geometry.

\subsection{Comparison with numerical simulations}

Here, we briefly compare our results with other available studies. First, Benson et al. (2000) presented a semianalytic model of galaxy formation to compute the kinetic SZ effect (within a $\Lambda \mathrm{CDM}$ cosmology). They roughly get the same amplitude $C_{l} \sim 10^{-13}$ at $l \sim 10^{4}$ and they also find that at low wavenumbers $\left(l \lesssim 10^{3}\right)$ most of the power is provided by density fluctuations and the clustering of ionization sources. However, because of numerical resolution limitations they get a sharp drop at $l \sim 210^{4}$ while we obtained a plateau for $C_{l}$ up to $l \sim 10^{6}$. Note that our model is entirely analytic, although the scaling function $H(x)$ which enters the multiplicity functions in (17) is obtained from a fit to $N$-body simulations, so that we have no resolution limitations (we are simply limited by the approximations involved in our model).

The secondary anisotropies produced by inhomogeneous reionization which we study in this article have also been computed by means of numerical simulations in Bruscoli et al. (2000), Gnedin \& Jaffe (2000) and Springel et al. (2000), using different cosmologies and astrophysical models. These authors find a broad maximum for the power-spectra $C_{l}$ and $S_{l}$ of $C_{l} \sim 10^{-13}-10^{-12}$ around $l \sim 10^{4}$. In particular, Springel et al. (2000) get a slowly decreasing plateau down to $l \sim 500$ (below this scale they are limited by finite box size effects while both other numerical studies are restricted to $l \gtrsim 10^{4}$ ). This behaviour agrees with our results (see the curve $S_{l, \mathrm{u}}$ in Fig. 5) since these authors use a simple toy model without including galaxy formation and radiative processes so that they miss the additional power due to the correlation of ionizing sources. Moreover, the drop we get for $C_{l}$ at $l \lesssim 300$, where we recover a white noise power spectrum, is beyond the range of these numerical simulations.

On small scales, Gnedin \& Jaffe (2000) (with the highest resolution) find a plateau at $C_{l} \sim 10^{-13}$ which slowly decreases up to their resolution limit at $l \sim 10^{6}$. This again roughly agrees with our predictions, although we rather obtain a slight increase of the total power with $l$ in this range. Note that these numerical simulations are restricted to $z>4$ and these authors estimate the missing signal by a simple extrapolation (i.e. they multiply their output by a factor 1.25). However, as we discussed in Sect. 3.5 the redshift distribution of the kinetic SZ effect depends on the angular scale one considers. Thus, large wavenumbers $\left(l \sim 10^{5}\right)$, which probe high density fluctuations, are more sensitive to low $z$ than large scales $\left(l \sim 10^{3}\right)$, which probe the inhomogeneous pattern of reionization and where most of the signal comes from epochs close to the reionization redshift $z_{\mathrm{ri}}$. This could explain the small difference between both predictions for the slope of this plateau. We can expect that with a higher resolution these authors would also recover a sharp cutoff at $l \gtrsim 10^{6}$ for $S_{l}$ (note that this drop is not readily apparent if one only computes the oscillatory spectrum $C_{l}$, see Fig. 4). These two behaviours are recovered by Bruscoli et al. (2000) at $l \sim 210^{5}$, but this smaller value for the location of the transition might be due to the finite numerical resolution. Since this scale is directly related to the size of virialized halos we can expect our result to be rather robust (in fact we would even expect some power at slightly smaller scales due to the collapse of baryons after they cool and to the substructures within halos, which would improve the agreement of our predictions with the results of Gnedin \& Jaffe (2000).

Bruscoli et al. (2000) also display the angular correlation function $C(\theta)$. It reaches a plateau $C(\theta) \sim 510^{-12}$ for $\theta<10^{-5} \mathrm{rad}$ and it shows oscillations for $\theta>210^{-4} \mathrm{rad}$. Thus, the amplitude of the signal they get is larger than our predictions (as for $C_{l}$ ). This could be due in part to their higher reionization redshift, see (49). Moreover, Gnedin \& Jaffe (2000) argue that those authors overestimate the SZ effect by a factor $3-10$ because of the uncorrected periodicity of the simulations. On the other hand, we obtain more large-scale power since in our model the cutoff of $C(\theta)$ only appears for $\theta \gtrsim 10^{-3} \mathrm{rad}$. On these large scales, secondary CMB anisotropies are generated by the cross-correlations of ionized bubbles. Hence this difference between both predictions may also be related to our smaller value for $z_{\text {ri }}$ since in our case at reionization structure formation is more advanced and the correlation length of the matter density field (hence of the radiation sources) is larger. Moreover, these large scales are not adequately resolved by these numerical simulations, as shown by their results for $C_{l}$ which are restricted to $l \gtrsim 10^{4}$.

Finally, we note that the independent study by Gnedin \& Jaffe (2000) also finds that the signal is dominated by the contribution from high-density ionized regions rather than from the patchy pattern of reionization (for $l>10^{4}$ ). This agrees with our results. Note that we find in addition that the inhomogeneous pattern of reionization plays an important role at larger scales $\left(l \sim 10^{3}\right)$ but this is beyond the range of these simulations. Thus, the agreement of our predictions with these various numerical studies, which use different cosmologies and astrophysical models, 
appears quite reasonable. Note that those numerical works do not include quasar formation models.

\section{Conclusion}

In this article, we have presented an analytic model (based on our previous work which described structure formation processes and the reionization history of the universe) which allows us to compute the secondary CMB anisotropies generated by the kinetic Sunyaev-Zel'dovich effect. This model includes a consistent description of galaxies, quasars and matter density fluctuations.

We have found that the contribution due to patchy reionization is negligible except at very large scales $(\theta \gtrsim$ $\left.10^{-3} \mathrm{rad}\right)$ and small wavenumbers $\left(l \lesssim 10^{3}\right)$. Over this range, which corresponds to scales larger than the typical size of HII regions, the signal actually comes from the cross-correlation of ionized bubbles, induced by the correlations of the rare radiation sources. On smaller scales, the IGM contribution is governed by the fluctuations of the matter density field itself. However, over this range the secondary anisotropies should be dominated by the contribution from galactic halos, which are characterized by smaller scales than the IGM (and larger densities). This leads to a cutoff of the power-spectrum $l(l+1) C_{l}$ at a large wavenumber $l \sim 10^{6}$. On the other hand, at low wavenumbers $l<10^{3}$ we recover a white noise power-spectrum. This very extended range of wavenumbers $10^{3}<l<10^{6}$ is close to the limitations of current numerical simulations. Thus, observations of these secondary CMB anisotropies should mainly probe the correlation properties of the underlying matter density field, through the correlations of the HII regions and the small-scale density fluctuations. We also found that the "local average" $S_{l}$ of the powerspectrum should be a more convenient tool than $C_{l}$.

Some comments are in order about the detectability of the effects described in this paper. First, we notice that in the range $10^{2} \lesssim l \lesssim 10^{4}$ the power predicted by our model (relative to primary anisotropies) is comparable to the one found by Knox et al. (1998). Following their conclusions, we infer that this signal, if not taken into account correctly, might introduce a small bias in the determination of cosmological parameters from future experiments like MAP (http://map.gsfc.nasa.gov) and particullarly Planck (http://astro.estec.esa.nl/SA-general/Projects/ Planck/). Second, although the range of $l$ where our model produces most of the power $\left(10^{4} \lesssim l \lesssim 10^{6}\right)$ is likely to be out of reach for MAP and Planck, future mm-wavelength interferometers, such as ALMA (http://www.mma.nrao.edu) may have the right sensitivity $\left(\sim 2 \mu \mathrm{K} \mathrm{rms}\right.$ for a $1^{\prime}$ beam in one hour $)$ and the right resolution $\left(<2^{\prime}\right)$ to be able to measure such a signal. Indeed, although the amplitude of the secondary anisotropies which we obtain is somewhat lower than the sensitivity of ALMA, a larger normalization of the power-spectrum (for the SCDM case we used $\sigma_{8}=0.5$ while a COBE normalization would give $\sigma_{8} \sim 1.4$ ) or a larger reionization redshift would push the signal into the range of detectability.

We noticed that the redshift distribution of the contributions to these secondary CMB anisotropies is rather broad. Thus, for the angular correlation from the IGM we get $7.5<z<10$ with a sharp cutoff at the reionization redshift $z_{\mathrm{ri}}=6.8$, when the "patchy pattern" of hydrogen ionization disappears. However, some small-scale anisotropies are still produced at lower redshifts. The redshift distributions of the contributions from galactic halos are even broader, we typically get $0<z<7$, and show no strong feature at $z_{\text {ri }}$. Since the total signal should be dominated by the contribution from these collapsed objects for a large range of wavenumbers $\left(l \gtrsim 10^{4}\right)$ this implies that one should not assume that most of the secondary CMB anisotropies are generated during a small redshift interval $\delta z$ around the reionization redshift $z_{\mathrm{ri}}$.

Next, as expected we have found that within our scenario ionized bubbles produced by quasars are larger than those built by galaxies. This implies that the "patchy patterns" of the HII regions associated with QSOs and stars are different. However, since the total signal is dominated by the correlations of the matter density field, and not by the size of the ionized bubbles, it is similar for both radiation sources (which also have similar correlation properties). Hence, unfortunately one cannot distinguish a quasar-driven reionization process from a galaxy-driven reionization history, using the $\mathrm{CMB}$ anisotropies.

Finally, we have checked that our predictions apply both for an open cosmology and for a critical density universe. Thus, our conclusions do not depend on the cosmological scenario and can be extended to low-density flat models. However, the amplitude of the anisotropies is larger for the low-density universe because of the higher reionization redshift.

\section{References}

Abel, T., Norman, M. L., \& Madau, P. 1999, ApJ, 523, 66

Aghanim, N., Désert, F. X., Puget, J. L., \& Gispert, R. 1996, A\&A, 311, 1

Balian, R., \& Schaeffer, R. 1989, A\&A, 220, 1

Benson, A. J., Nusser, A., Sugiyama, N., \& Lacey, C. G. 2000, to appear in MNRAS [astro-ph 0002457]

Bernardeau, F., \& Schaeffer, R. 1992, A\&A, 255, 1

Bernardeau, F., \& Schaeffer, R. 1999, A\&A, 349, 697

Bruscoli, M., Ferrara, A., Fabbri, R., \& Ciardi, B. 2000, submitted to MNRAS [astro-ph 9911467]

Davis, M., Efstathiou, G. P., Frenk, C. S., \& White, S. D. M. 1985, ApJ, 292, 371

Gnedin, N. Y. 2000, accepted by ApJ [astro-ph 9909383]

Gnedin, N. Y., \& Jaffe, A. 2000, submitted to ApJ [astro-ph 0008469]

Gradshteyn, I. S., \& Ryzhik, I. M. 1965, Table of integrals, series and products, fifth edition (Academic Press)

Groth, E. J., Juskiewicz, R., \& Ostriker, J. P. 1989, ApJ, 346, 558

Gruzinov, A., \& Hu, W. 1998, ApJ, 508, 435

Kaiser, N. 1984, ApJ, 282, 374 
Knox, L., Scoccimarro, R., \& Dodelson, S. 1998, Phys. Rev. Lett., 81, 2004

Krauss, L., \& Turner, M. S. 1995, Gen. Rel. Grav., 27, 1137

Madau, P. 2000, submitted to the Philosophical Transactions of the Royal Society A [astro-ph 0003096]

Munshi, D., Coles, P., \& Melott, A. L. 1999, MNRAS, 310, 892

Ostriker, J. P., \& Steinhardt, P. J. 1995, Nature, 377, 600

Ostriker, J. P., \& Vishniac, E. T. 1986, ApJ, 306, L51

Peebles, P. J. E. 1980, The large scale structure of the Universe, (Princeton University Press)

Rees, M. J. 1984, ARA\&A, 22, 471

Seljak, U., \& Zaldarriaga, M. 1996, ApJ, 469, 437

Springel, V., White, M., \& Hernquist, L. 2000, submitted to
ApJL [astro-ph 0008133]

Sunyaev, R. A. 1978, in Large-scale structure of the Universe, ed. M. S. Longair, \& J. Einasto (Dordrecht: Reidel), 393

Sunyaev, R. A., \& Zel'dovich, Y. B. 1980, MNRAS, 190, 413

Tumlinson, J., \& Shull, J. M. 2000, ApJ, 528, L65

Valageas, P., \& Schaeffer, R. 1997, A\&A, 328, 435

Valageas, P., \& Schaeffer, R. 1999, A\&A, 345, 329

Valageas, P., \& Silk, J. 1999a, A\&A, 347, 1

Valageas, P., \& Silk, J. 1999b, A\&A, 350, 725

Valageas, P., Schaeffer, R., \& Silk, J. 1999, A\&A, 345, 691

Valageas, P., \& Schaeffer, R. 2000, A\&A, 359, 821

Valageas, P., Schaeffer, R., \& Silk, J. 2000, accepted by A\&A [astro-ph 0001207] 